\title{
A model with interaction of dark components and recent observational data
}

\author{
Supriya Pan ${ }^{1,}$, and German S. Sharov, \\ ${ }^{1}$ Department of Physical Sciences, Indian Institute of Science Education \\ and Research Kolkata, Mohanpur-741246, West Bengal, India \\ ${ }^{2}$ Tver State University, 170002, Sadovyj per. 35, Tver, Russia
}

\begin{abstract}
In the proposed model with interaction between dark energy and dark matter, we consider cosmological scenarios with different equations of state $\left(w_{d}\right)$ for dark energy. For both constant and variable equation of state, we analyze solutions for dark energy and dark matter in seven variants of the model. We investigate exact analytic solutions for $w_{d}=$ constant equation of state, and several variants of the model for variable $w_{d}$. These scenarios are tested with the current astronomical data from Type Ia Supernovae, baryon acoustic oscillations, Hubble parameter $H(z)$ and the cosmic microwave background radiation. Finally, we make a statistical comparison of our interacting model with $\Lambda$ CDM as well as with some other well known non-interacting cosmological models.
\end{abstract}

PACS numbers: 98.80.-k, 95.35.+d, 95.36.+x

Keywords: cosmological parameters; dark energy; dark matter; observations; theory

The current accelerated expansion of the universe ascertained from several astronomical sources [1/7] is one of the appealing fields of research in modern cosmology. To explain such accelerated expansion, either one needs some hypothetical dark energy fluid in the framework of general relativity or claims direct modifications into the gravitational sector leading to several modified gravity theories. However, a large number of observational data favor the existence of $\Lambda$ CDM-cosmology where the cosmological constant fluid acts as a hypothetical dark energy fluid occupying almost two third of the total energy density of the universe and the cold dark matter (CDM) fluid is responsible for the structure formation of the universe. Nevertheless, the cosmological constant problem [8] and the cosmic coincidence problem [9] associated with the $\Lambda \mathrm{CDM}$ cosmology lead to several alternative descriptions aiming to decode the dynamics of the universe, such as the dynamical dark energy models, modified gravity models, and some others. For a comprehensive discussions on such models and their effects on the dynamics of the universe, we refer [10.

Amongst several dark energy and modified gravity models, interacting dark energy models have gained significant attention due to having a reasonable explanation to the cosmic coincidence problem. A number of investigations in this direction have been performed during last couple of years [11 28]. Recently, a series of analysis shows that the current observational data prefer a nonzero interaction in the dark sector 28,33 . On the other hand, the dark sector's interaction may alleviate the current tension on local value of the Hubble constant and on $\sigma_{8}$ as well [31, 32, 34 36. Further, it has been discussed that some appropriate choice of an interaction between the dark components can influence effectively on the perturbation analysis which results in quite no-

\footnotetext{
*Electronic address: span@iiserkol.ac.in
}

${ }^{\dagger}$ Electronic address: Sharov.GS@tversu.ru table differences in the lowest multipoles of the cosmic microwave background spectrum [37, 38. And sometimes it is possible to address the phantom universe [3944 by a nonzero coupling in the dark sector [30, 45, 46. For a review on interacting dark energy and its several consequences see 447, 48. Therefore, based on the above arguments it is evident that the possibility of mutual interaction in the dark sectors may serve as an alternative description to understand the dynamical evolution of the universe.

In the present work we thus consider the scenario of the universe where DE is interacting with CDM through a nongravitational interaction $Q$ which in general is an arbitrary function of the cosmological variables. We consider a particular model of $Q$ [49] which is the linear combination of DE and CDM. We study this interaction model both for constant and variable equation of state in DE and provide an updated constraints on the model parameters using the latest observational data from Union 2.1, Hubble parameter measurements, baryon acoustic oscillation (BAO) data and the cosmic microwave background radiation (CMB). Finally, we make a statistical comparison of the present interacting model with $\Lambda \mathrm{CDM}$ as well as other known non-interacting cosmological models (Sect. 4).

The paper is organized as follows: In section 1, we describe the basic equations of interacting dynamics in the non-flat FLRW model. For the linear interaction between DE and CDM, section 2 presents the analytic solutions for $w_{d}=$ const, in the EoS of DE and 5 variants of the model for variable $w_{d}$. In section 3 , we shortly discuss different joint data analysis techniques and the results of their application to the considered several variants of our model. In section 4 we compare this model with other popular models in describing the same observational data. Finally, in section 5, we summarize the results of the work. Let us note that any subindex "0" attached to any quantity refers its value at present time. 


\section{INTERACTING DYNAMICS IN FLRW UNIVERSE}

Let us consider the homonegeous and isotropic universe characterized by the Friedmann-LemmitreRobertson-Walker (FLRW) line element

$\mathrm{ds}^{2}=-\mathrm{d} t^{2}+a^{2}(t)\left[\mathrm{d} r^{2} /\left(1-k r^{2}\right)+r^{2}\left(\mathrm{~d} \theta^{2}+\sin ^{2} \theta \mathrm{d} \phi^{2}\right)\right]$,

where $a(t)$ is the scale factor of the universe and $k$ is the spatial curvature which represents a flat, open and closed universe respectively for $k=0,-1$, and 1 . In such a background, the first Friedmann equation can be written as

$$
H^{2}+\frac{k}{a^{2}}=\frac{8 \pi G}{3} \rho,
$$

where $H=\dot{a} / a$, is the Hubble rate of the FLRW universe; $\rho$ is the total energy density of the universe which is the mixture of baryons, cold dark matter and dark energy, i.e. $\rho=\rho_{b}+\rho_{d m}+\rho_{d}$, where $\rho_{b}, \rho_{d m}, \rho_{d}$ are respectively the energy densities of baryons, CDM and DE. We further assume that CDM and DE are interacting with each other while baryons do not take part in the interation. The energy conservation equation for the total fluid follows

$$
\dot{\rho}+3 \frac{\dot{a}}{a}(p+\rho)=0 .
$$

Since only CDM and DE interact with each other but baryons do not interact, thus, the evolution for baryons follows $\dot{\rho}_{b}+3 H \rho_{b}=0 \Longrightarrow \rho_{b}=\rho_{b 0} a^{-3}$, while the evolution equations for CDM and DE read

$$
\begin{aligned}
\dot{\rho}_{d m}+3 H \rho_{d m} & =Q, \\
\dot{\rho}_{d}+3 H\left(1+w_{d}\right) \rho_{d} & =-Q,
\end{aligned}
$$

where $Q$ is the interaction function between the dark sectors. Physically, the interaction is charaterized by some energy flow between the sectors interacting with each other. A positive interaction (i.e. $Q>0$ ) implies the flow of energy from DE to CDM while its negative value denotes the energy flow in the opposite direction. Now, introducing the total energy density of CDM and DE as $\rho_{T}=\rho_{d m}+\rho_{d}$, it is easy to see that the combination of eqns. (3) and (4) turns into

$$
\dot{\rho}_{T}+3 \frac{\dot{a}}{a}\left(p_{T}+\rho_{T}\right)=0 .
$$

Now, using Eq. (5), one can express $\rho_{d}$ and $\rho_{d m}$ as follows:

$$
\begin{aligned}
\rho_{d} & =-\frac{\rho_{T}+\rho_{T}^{\prime}}{w_{d}}, \\
\rho_{d m} & =\frac{\rho_{T}^{\prime}+\left(1+w_{d}\right) \rho_{T}}{w_{d}} .
\end{aligned}
$$

Here primes denote derivatives with respect to the variable $x=3 \ln \left(a / a_{0}\right)=3 \ln a$ (We set $a_{0}=1$ as an usual practice and there is no loss of generality). Thus, once
$\rho_{T}$ is determined, the evolution equations for CDM and DE can be understood. However, in the present study we shall concentrate on an interaction function which is the linear combination of the energy densities of CDM and DE. In what follows in the next section we discuss the interacting scenarios both for constant and dynamical equation of state in DE.

\section{VARIANTS OF THE MODEL}

We introduce the following interaction [19, 49]

$$
Q=3 \lambda_{m} H \rho_{d m}+3 \lambda_{d} H \rho_{d}
$$

where $\lambda_{m}, \lambda_{d}$ are the coupling parameters that denote the strength (with their magnitudes) and the direction of energy flow (with their signs) between the interacting sectors. Due to the expression (8) the conservation equations (3) and (4) are modified, and finally, we get the following second order differential equation:

$$
\begin{aligned}
\rho_{T}^{\prime \prime} & +\left(2+w_{d}+\lambda_{d}-\lambda_{m}-\frac{w_{d}^{\prime}}{w_{d}}\right) \rho_{T}^{\prime} \\
& +\left[\left(1+w_{d}\right)\left(1-\lambda_{m}\right)+\lambda_{d}-\frac{w_{d}^{\prime}}{w_{d}}\right] \rho_{T}=0
\end{aligned}
$$

which is the master equation to determine the evolution of CDM and DE. Let us proceed with two different possibilities with the equation of state in DE, namely when it is either constant or dynamical with the cosmic evolution.

\subsection{The case for constant EoS in DE}

If $w_{d}=$ constant, the solution of the differential equation (9) becomes 22]

$$
\rho_{T}=\rho_{1} a^{3 m_{+}}+\rho_{2} a^{3 m_{-}},
$$

where $\rho_{1}, \rho_{2}$ are integration constants, $m_{+}, m_{-}$are

$m_{ \pm}=\frac{\lambda_{m}-w_{d}-\lambda_{d}-2 \pm \sqrt{\left(\lambda_{m}+w_{d}+\lambda_{d}\right)^{2}-4 \lambda_{m} \lambda_{d}}}{2}$.

One can see that for this case, the Hubble function (1) takes an analytic form leading to

$$
H^{2}=\frac{8 \pi G}{3}\left[\rho_{b 0} a^{-3}+\rho_{1} a^{3 m_{+}}+\rho_{2} a^{3 m_{-}}\right]-\frac{k}{a^{2}}
$$

Now, using 10 , we have the explicit analytic solutions for dust and dark energy as follows:

$$
\begin{aligned}
\rho_{d m} & =\rho_{1} \frac{w_{d}+1+m_{+}}{w_{d}} a^{3 m_{+}}+\rho_{2} \frac{w_{d}+1+m_{-}}{w_{d}} a^{3 m_{-}}, \\
\rho_{d} & =-\frac{\rho_{1}\left(1+m_{+}\right) a^{3 m_{+}}+\rho_{2}\left(1+m_{-}\right) a^{3 m_{-}}}{w_{d}} .
\end{aligned}
$$


We mention that in Ref. 49 the analytic solution for this particular linear interaction was discussed assuming that the magnitudes of both coupling parameters are very small, that means, the product $\lambda_{m} \lambda_{d}$ was excluded and the cosmlogical scenario wer analyzed for the solution with $m_{+}=-\left(1-\lambda_{m}\right), m_{-}=-\left(1+\lambda_{d}+w_{d}\right)$. Certainly, a detailed analysis with no such restriction is worth investigating. Moreover, the analysis of this model was performed with 194 Supernovae Type Ia data from [50, 51, which needs to be updated with the latest observational data. Thus, in comparison with the previous study, the present one has two fold importance: (i) the solution (10) for general $\left(m_{+}, m_{-}\right)$completes the study without any information loss, and (ii) here we employ the current observational data which provide better observational constraints on all model parameters. Thus, under (i) and (ii), the present analytic interacting dark energy model could produce some interesting information about this interacting dark energy-dark matter model while constraining it by recent observational data sets.

Further, the usual density parameters for dark matter $\left(\Omega_{d m 0}\right)$ and dark energy $\left(\Omega_{d 0}\right)$ in terms of the density parameters for the equivalent two fluids $\Omega_{1}$ and $\Omega_{2}$ are given by

$$
\begin{aligned}
\Omega_{d m 0} & =\Omega_{1} \frac{w_{d}+1+m_{+}}{w_{d}}+\Omega_{2} \frac{w_{d}+1+m_{-}}{w_{d}}, \\
\Omega_{d 0} & =-\frac{\Omega_{1}\left(1+m_{+}\right)+\Omega_{2}\left(1+m_{-}\right)}{w_{d}},
\end{aligned}
$$

where $\Omega_{i}=8 \pi G \rho_{i} / 3 H_{0}^{2}$. The values $\Omega_{1}, \Omega_{2}$ can be expressed by using the above two equations (11), 12), their consequence $\Omega_{d m 0}+\Omega_{d 0}=\Omega_{1}+\Omega_{2}$ and the equality

$$
\Omega_{d m 0}+\Omega_{d 0}+\Omega_{b 0}+\Omega_{k}=\Omega_{1}+\Omega_{2}+\Omega_{b 0}+\Omega_{k}=1,
$$

results in from Eq. (1) at the present time $t=t_{0}$. Here $\Omega_{b 0}=\Omega_{b}\left(t_{0}\right), \Omega_{k}=-k /\left(a_{0} H_{0}\right)^{2}$. In particular,

$$
\Omega_{1}=\frac{w_{d} \Omega_{d m 0}-\left(1+w_{d}+m_{-}\right)\left(1-\Omega_{b 0}-\Omega_{k}\right)}{m_{+}-m_{-}} .
$$

Also, we note that the total density parameter for matter is, $\Omega_{m 0}=\Omega_{d m 0}+\Omega_{b 0}$. In Sect. 3 (see Fig. 1) we investigate how solutions $(10)$ describe the observational data for Type Ia supernovae, baryon acoustic oscillations, for the Hubble parameter $H(z)$ and CMB.

\subsection{Variable EoS in DE}

In this section we focus on the interacting models where the EoS in DE, $w_{d}$, is dynamical. There are several interacting dark energy models with possibility of variable $\mathrm{EoS}$ in $\mathrm{DE}$, where reasonable attention has been paid to observational data. In Ref. [52, the authors investigated an interacting scenario for $Q=3 H \lambda_{m} \rho_{m}$ with Chevallier-Polarski-Linder (CPL) parametrization [53, 54] as the equation of state in DE. Also, in Ref.
[18 the authors studied the present linear interaction (8) with CPL parametrization but with very old data (182 Gold Type Ia Supernoave data [55]). Thus, considering the linear interaction (8) in our discussion, we aim to investigate the interacting dynamics between $\mathrm{CDM}$ an $\mathrm{DE}$ with some new variable equations of state in $w_{d}$ including CPL [53, 54] and linear parametrization [56 58] by Union 2.1 compilation [59] along with Hubble parameter measurements, baryon acoustic oscillation and CMB data.

Let us first begin our analysis with the following generalized ansatz

$$
\frac{w_{d}^{\prime}}{w_{d}}=\alpha w_{d}+\beta,
$$

where $\alpha, \beta$ are real numbers. We note that the EoS (14) is the generalized version of the variable EoS of DE presented in [49]. The solution of Eq. (14) is

$$
w_{d}=\left[\left(\frac{1}{w_{d 0}}+\frac{\alpha}{\beta}\right) a^{-3 \beta}-\frac{\alpha}{\beta}\right]^{-1} .
$$

In particular, we consider the following partial cases

$$
\begin{aligned}
\text { Ansatz I: } & \alpha=0, \quad w_{d}=w_{d 0} a^{3 \beta} \\
\text { Ansatz II: } & \beta=0, \quad w_{d}=\frac{w_{d 0}}{\left(1-3 \alpha w_{d 0} \ln a\right)} .
\end{aligned}
$$

We also consider separately the following ansatz:

$$
\text { Ansatz III: } \quad \alpha=1,
$$

which is attractive, because in this case under the condition $\lambda_{m}=0$ coefficients of equation (9) become constant, and its general solution has the simple form [49]

$$
\rho_{T}=\tilde{\rho}_{1} a^{-3}+\tilde{\rho}_{2} a^{3(n-1)},
$$

where $n=\beta-\lambda_{d}=$ const, and $\tilde{\rho}_{1}>0, \tilde{\rho}_{2}>0$ are integration constants.

Moreover, we also consider two more interacting scenarios when the EoS of DE obeys the Chevallier-PolarskiLinder (CPL) parametrization [53, 54]

$$
\text { Ansatz IV: } \quad w_{d}(z)=w_{d 0}+w_{1} \frac{z}{1+z},
$$

and the linear parametrization $[56 \sqrt[58]{58}$

$$
\text { Ansatz V: } \quad w_{d}(z)=w_{d 0}+w_{1} z .
$$

Here in both 20], 21), $w_{d 0}$, and $w_{1}=d w_{d}(z) / d z$ at $z=0$ are two free parameters to be constrained by the observational data. The dependencies of $w_{d}(z)$ in $(20)$ and (21) are alternative to (15).

\section{JOINT ANALYSIS}

In order to constrain the proposed models with recent observational data, we use $N_{S N}=580$ data points 
for Type Ia supernovae from Union 2.1 [59], $N_{H}=39$ observed Hubble data points [60 75] and $N_{B A O}=17$ baryon acoustic oscillation data 66 84, and finally the cosmic microwave background radiation $(\mathrm{CMB})$ in the form 85 .

Our analysis follows the likelihood $\mathcal{L} \propto \exp \left(-\chi^{2} / 2\right)$ where $\chi^{2}=\sum_{i} \chi_{i}^{2}$ ( $i$ runs over the all data sets employed in the analysis). We calculate the best-fitted values of the free model parameters with their corresponding uncertainties from the minimization of the $\chi^{2}$ function. We use two different combined analysis with the likelihoods $\mathcal{L}_{\Sigma} \propto \exp \left(-\chi_{\Sigma}^{2} / 2\right), \mathcal{L}_{\text {tot }} \propto \exp \left(-\chi_{\text {tot }}^{2} / 2\right)$, where

$$
\begin{aligned}
\chi_{\Sigma}^{2} & =\chi_{S N}^{2}+\chi_{H}^{2}+\chi_{B A O}^{2}, \\
\chi_{\text {tot }}^{2} & =\chi_{S N}^{2}+\chi_{H}^{2}+\chi_{B A O}^{2}+\chi_{C M B}^{2} .
\end{aligned}
$$

In the next subsections, we shall shortly describe different data sets and the corresponding $\chi^{2}$ functions.

\subsection{Union 2.1 data points}

Type Ia Supernovae are the first indication for existence of some dark energy in our Universe [1, 2. The observable quantities from a Type Ia supernova (SN Ia) are its redshift $z$ and its apparent magnitude $m_{\text {obs }}$, but in the survey [59] values $m_{o b s}$ are recalculated into distance modulus

$$
\mu_{o b s}=m_{o b s}(z)-M+\bar{\alpha} x_{1}-\bar{\beta} c+\bar{\delta} P .
$$

Here, additive terms include the SN Ia absolute magnitude $M$ and corrections connected with deviations from mean values of lightcurve shape $\left(x_{1}\right)$, SN Ia color $(c)$ and mass of a host galaxy (the factor $P$ ). The parameters $M, \bar{\alpha}, \bar{\beta}$ and $\bar{\delta}$ are considered in Ref. [59] as nuisance parameters, and they are fitted simultaneously with $H_{0}$ and other cosmological parameters in the flat $\Lambda$ CDM model. This approach is usual in SN Ia analysis [86 88. So values (24) in Ref. 59] may have a model dependent additive term (a systematic error) with concealed dependence on $H_{0}$ and other model parameters.

We have to keep in mind this fact, when we compare the observable values (24) from Ref. [59] with theoretical values of distance modulus, corresponding to redshift $z$ :

$$
\mu_{t h}(z)=5 \log _{10}\left(\frac{D_{L}(z)}{10 \mathrm{pc}}\right)=5 \log _{10} \frac{H_{0} D_{L}}{c}+\mu_{0} .
$$

Here, $\mu_{0}=42.384-5 \log _{10} h, D_{L}(z)$ is the luminosity distance [1, 86.

$$
D_{L}(z)=\frac{c(1+z)}{H_{0}} S_{k}\left(H_{0} \int_{0}^{z} \frac{d \tilde{z}}{H(\tilde{z})}\right)
$$

with

$$
S_{k}(x)= \begin{cases}\sinh \left(x \sqrt{\Omega_{k}}\right) / \sqrt{\Omega_{k}}, & \Omega_{k}>0 \\ x, & \Omega_{k}=0 \\ \sin \left(x \sqrt{\left|\Omega_{k}\right|}\right) / \sqrt{\left|\Omega_{k}\right|}, & \Omega_{k}<0\end{cases}
$$

The value $H_{0} D_{L} / c$ in Eq. 25 is the Hubble free luminosity distance (for the majority of cosmological models) and only the term $\mu_{0}$ [86 depend on the Hubble constant $H_{0}$ or $h=H_{0} / 100 \mathrm{~km} \mathrm{~s}^{-1} \mathrm{Mpc}^{-1}$.

For any cosmological model, we fix its model parameters $\theta_{1}, \theta_{2}, \ldots$, calculate functions $a(t), z=a^{-1}-1$, $H(z)$, the integral 26 , and hence this model predicts theoretical values $D_{L}^{t h}$ or $\mu_{t h}$ for the modulus 25). To compare these theoretical values with the observational data $z_{i}$ and $\mu_{o b s}\left(z_{i}\right)$ [59] we use the $580 \times 580$ covariance matrix $C_{S N}$ from Ref. 59 and the function

$$
\tilde{\chi}_{S N}^{2}\left(\theta_{1}, \ldots\right)=\sum_{i, j=1}^{N_{S N}} \Delta \mu_{i}\left(C_{S N}^{-1}\right)_{i j} \Delta \mu_{j}
$$

where $\Delta \mu_{i}=\mu_{t h}\left(z_{i}, \theta_{1}, \ldots\right)-\mu_{o b s}\left(z_{i}\right)$.

To exclude the possible systematic errors in $\mu_{o b s}$ mentioned above, we follow the marginalization procedure, suggested in Ref. [86, and consider below the minimum of the sum 27 over $H_{0}$ (or over $\mu_{0}$ )

$$
\begin{aligned}
& \chi_{S N}^{2}=\min _{\mu_{0}} \tilde{\chi}_{S N}^{2}=\left.\tilde{\chi}_{S N}^{2}\right|_{\mu_{0}=0}-\frac{B^{2}}{C}, \\
& B=\sum_{i, j=1}^{N_{S N}}\left(\Delta \mu_{i}-\mu_{0}\right)\left(C_{S N}^{-1}\right)_{i j}, \quad C=\sum_{i, j=1}^{N_{S N}}\left(C_{S N}^{-1}\right)_{i j} .
\end{aligned}
$$

In this paper, for all models we use the marginalized function (28) to describe the SNe Ia data [59].

\subsection{Hubble parameter data}

The Hubble parameter $H$ at some certain redshift $z$ can be measured from differential ages of galaxies 60 65. with using the following formula:

$$
H(z)=\frac{\dot{a}}{a}=-\frac{1}{1+z} \frac{d z}{d t},
$$

In addition, estimations of $H(z)$ may be extracted from line-of-sight BAO data $66-75]$.

In this analysis we use $N_{H}=39$ observed Hubble parameter values 6075 in the range $0.070 \leq z \leq 2.36$, which are listed in Table (I). The corresponding $\chi_{H}^{2}$ is defined as

$$
\chi_{H}^{2}=\sum_{i=1}^{N_{H}}\left[\frac{H_{o b s}\left(z_{i}\right)-H_{t h}\left(z_{i}, \theta_{j}\right)}{\sigma_{H, i}}\right]^{2} .
$$

\subsection{BAO data}

Observational data, connected with baryon acoustic oscillations (BAO), include the distance 6]

$$
D_{V}(z)=\left[\frac{c z D_{L}^{2}(z)}{(1+z)^{2} H(z)}\right]^{1 / 3}
$$




\begin{tabular}{|c|c|c|c|c|c|c|c|}
\hline \begin{tabular}{l|}
$z$ \\
\end{tabular} & $H_{o b s}(z)$ & $\sigma_{H}$ & References & $z$ & $H_{o b s}(z)$ & $\sigma_{H}$ & References \\
\hline 0.070 & \begin{tabular}{|l|}
69 \\
\end{tabular} & 19.6 & 63 & 0.570 & \begin{tabular}{|l|}
96.8 \\
\end{tabular} & 3.4 & 72 \\
\hline 0.090 & 69 & 12 & 60 & 0.593 & 104 & 13 & 62 \\
\hline 0.120 & 68.6 & 26.2 & 63 & 0.600 & 87.9 & 6.1 & 67 \\
\hline 0.170 & 83 & 8 & 60 & 0.680 & 92 & 8 & 62 \\
\hline 0.179 & 75 & 4 & 62 & 0.730 & 97.3 & 7.0 & 67 \\
\hline 0.199 & 75 & 5 & 62 & 0.781 & 105 & 12 & 62 \\
\hline 0.200 & 72.9 & 29.6 & 63 & 0.875 & 125 & 17 & 62 \\
\hline 0.240 & 79.69 & 2.99 & 6 & 0.880 & 90 & 40 & 61 \\
\hline 0.270 & 77 & 14 & 60 & 0.900 & 117 & 23 & 60 \\
\hline 0.280 & 88.8 & 36.6 & 63 & 1.037 & 154 & 20 & 62 \\
\hline 0.300 & 81.7 & 6.22 & 73 & 1.300 & 168 & 17 & 60 \\
\hline 0.340 & 83.8 & 3.66 & 66 & 1.363 & 160 & 33.6 & 64 \\
\hline 0.350 & 82.7 & 9.1 & 69 & 1.430 & 177 & \begin{tabular}{|l|}
18 \\
\end{tabular} & 60 \\
\hline 0.352 & 83 & 14 & 62 & 1.530 & 140 & 14 & 60 \\
\hline 0.400 & 95 & 17 & 60 & 1.750 & 202 & 40 & 60 \\
\hline 0.429 & 91.8 & 5.3 & 65 & 1.965 & 186.5 & 50.4 & 64 \\
\hline 0.430 & 86.45 & 3.97 & 66 & 2.300 & 224 & 8.6 & 68 \\
\hline 0.440 & 82.6 & 7.8 & 67 & 2.340 & 222 & 8.5 & 75 \\
\hline 0.480 & 97 & 62 & 61 & 2.360 & 226 & 9.3 & 74 \\
\hline 0.570 & 87.6 & 7.8 & 70 & & & & \\
\hline
\end{tabular}

TABLE I: Hubble parameter values $H_{o b s}$ in $\mathrm{km} \mathrm{s}^{-1} \mathrm{Mpc}^{-1}$ at different redshifts $z$ with corresponding errors $\sigma_{H}$.

\begin{tabular}{||l|l|l|l|l|c|l||}
\hline$z$ & $d_{z}(z)$ & $\sigma_{d}$ & $A(z)$ & $\sigma_{A}$ & References & Survey \\
\hline 0.106 & 0.336 & 0.015 & 0.526 & 0.028 & {$[78,[84$} & 6dFGS \\
\hline 0.15 & 0.2232 & 0.0084 & - & - & {$[83$} & SDSS DR7 \\
\hline 0.20 & 0.1905 & 0.0061 & 0.488 & 0.016 & {$[76,[79]$} & SDSS DR7 \\
\hline 0.275 & 0.1390 & 0.0037 & - & - & {$[76]$} & SDSS DR7 \\
\hline 0.278 & 0.1394 & 0.0049 & - & - & {$[77]$} & SDSS DR7 \\
\hline 0.314 & 0.1239 & 0.0033 & - & - & {$[79$} & SDSS LRG \\
\hline 0.32 & 0.1181 & 0.0026 & - & - & {$[72$} & BOSS DR11 \\
\hline 0.35 & 0.1097 & 0.0036 & 0.484 & 0.016 & {$[76,[79$} & SDSS DR7 \\
\hline 0.35 & 0.1126 & 0.0022 & - & - & {$[80]$} & SDSS DR7 \\
\hline 0.35 & 0.1161 & 0.0146 & - & - & {$[69$} & SDSS DR7 \\
\hline 0.44 & 0.0916 & 0.0071 & 0.474 & 0.034 & {$[79$} & WiggleZ \\
\hline 0.57 & 0.0739 & 0.0043 & 0.436 & 0.017 & {$[70$} & SDSS DR9 \\
\hline 0.57 & 0.0726 & 0.0014 & - & - & {$[72$} & SDSS DR11 \\
\hline 0.60 & 0.0726 & 0.0034 & 0.442 & 0.020 & {$[79$} & WiggleZ \\
\hline 0.73 & 0.0592 & 0.0032 & 0.424 & 0.021 & {$[79$} & WiggleZ \\
\hline 2.34 & 0.0320 & 0.0021 & - & - & {$[75$} & BOSS DR11 \\
\hline 2.36 & 0.0329 & 0.0017 & - & - & {$[74$} & BOSS DR11 \\
\hline
\end{tabular}

TABLE II: Values of $d_{z}(z)=r_{s}\left(z_{d}\right) / D_{V}(z)$ and $A(z)$ with errors and references

and two measured values

$$
d_{z}(z)=\frac{r_{s}\left(z_{d}\right)}{D_{V}(z)}, \quad A(z)=\frac{H_{0} \sqrt{\Omega_{m 0}}}{c z} D_{V}(z) .
$$

Here $r_{s}\left(z_{d}\right)$ is sound horizon size at the end of the drag era $z_{d}$. In this paper we use the fitting formula from
Ref. 89

$$
r_{s}\left(z_{d}\right)=\frac{55.154 \exp \left[72.3\left(\Omega_{\nu} h^{2}+0.0006\right)^{2}\right]}{\left(\Omega_{m 0} h^{2}\right)^{0.25351}\left(\Omega_{b 0} h^{2}\right)^{0.12807}} \mathrm{Mpc} .
$$

Here dependence on neutrino contribution $\Omega_{\nu}$ is negligible for reasonable values $\sum m_{\nu} \leq 0.23 \mathrm{eV}$ [2] (below we 
suppose $\sum m_{\nu}=0.06 \mathrm{eV}[42,89]$ ).

Calculations with similar observational data and with the function (31) were made in Ref. [90, for the models: $\Lambda \mathrm{CDM}$, with generalized and modified Chaplygin gas and with quadratic equation of state (described below in Sect. 4. The best fitting value of $\Omega_{b 0}$ in Eq. (31)

$$
\Omega_{b 0}=0.044
$$

was obtained for the $\Lambda \mathrm{CDM}$ and appeared to be just the same for 3 other models in Ref. [90. One should note that the value (32) is connected with the formula 31. Calculations in Ref. 90 with the more simple fitting formula $r_{d}=\left(r_{d} h\right)_{f i d} \cdot h^{-1}$ for all 4 models demonstrated similar estimations of model parameters, but very weak dependence of them on $\Omega_{b 0}$. It is connected with similarity in properties of dark matter and baryons. Due to this reason we do not consider $\Omega_{b 0}$ as a free model parameter and fix it in the form $(32)$ for all models in this paper. The additional reason is necessity to minimize a number of free model parameters for considered scenarios.

To take into account all available BAO data 66, 67, 69? 84 for parameters (30), we consider in this paper $N_{B}=17$ data points for $d_{z}(z)$ and 7 data points for $A(z)$ presented in the Table II.

Measurements of $d_{z}(z)$ and $A(z)$ from Refs. 76, 79, in Table II are not independent. So the $\chi^{2}$ function for the values $(30)$ is

$$
\chi_{B A O}^{2}\left(\theta_{j}\right)=(\Delta d)^{T} C_{d}^{-1} \Delta d+(\Delta A)^{T} C_{A}^{-1} \Delta A,
$$

where $\Delta d=d_{z}\left(z_{i}\right)-d_{z}^{t h}, \Delta A=A\left(z_{i}\right)-A^{t h}$. The elements of covariance matrices $C_{d}^{-1}=\left\|c_{i j}^{d}\right\|, C_{A}^{-1}=\left\|c_{i j}^{A}\right\|$ in Eq. (33) are 76, 79, 84,

$$
\begin{array}{lll}
c_{33}^{d}=30124, & c_{38}^{d}=-17227, & c_{88}^{d}=86977, \\
c_{111}^{d}=24532.1, & c_{1114}^{d}=-25137.7, & c_{115}^{d}=12099.1, \\
c_{114}^{d}=134598.4, & c_{1415}^{d}=-64783.9, & c_{1515}^{d}=128837.6 ; \\
c_{1111}^{A}=1040.3, & c_{114}^{A}=-807.5, & c_{115}^{A}=336.8, \\
c_{1414}^{A}=3720.3, & c_{1415}^{A}=-1551.9, & c_{1515}^{A}=2914.9 .
\end{array}
$$

Here $c_{i j}=c_{j i}$, the remaining matrix elements are $c_{i j}=0$, if $i \neq j$, and $c_{i i}=1 / \sigma_{i}^{2}$.

\subsection{CMB data}

Cosmological data associated with the cosmic microwave background (CMB) radiation include parameters at the photon-decoupling epoch $z_{*}=1089.90 \pm 0.30$ [42, in particular, the comoving sound horizon $r_{s}\left(z_{*}\right)$ and the distance $D_{M}\left(z_{*}\right)=D_{L}\left(z_{*}\right) /\left(1+z_{*}\right)$ [85, 89]. In this paper we use the CMB parameters in the form [85]

$\mathbf{x}=\left(R, \ell_{A}, \omega_{b}\right)=\left(\sqrt{\Omega_{m}} \frac{H_{0} D_{M}\left(z_{*}\right)}{c}, \frac{\pi D_{M}\left(z_{*}\right)}{r_{s}\left(z_{*}\right)}, \Omega_{b} h^{2}\right)$.

In the corresponding $\chi^{2}$ function

$$
\chi_{C M B}^{2}=\Delta \mathbf{x} \cdot C_{C M B}^{-1}(\Delta \mathbf{x})^{T},
$$

we use the covariance matrix $C_{C M B}$ and the distance priors

$$
\Delta \mathrm{x}=\mathrm{x}-(1.7448,301.46,0.0224),
$$

from Ref. [85], which were derived from [42] data with free amplitude of the lensing power spectrum.

\subsection{Results for $w_{d}=$ constant.}

We investigated, how the $\chi^{2}$ functions $(22)$ and 23 ) depend on model parameters for different variants of the model, considered in Sect. 2. For the model with $w_{d}=$ const (equivalently, solution $(10)$ ), the constraints on the model parameters are presented in Table III and the corresponding plots are shown in Fig. 1. The first and third rows of panels in Fig. 11illustrate how the minimum of the sums 22) $\left(\min \chi_{\Sigma}^{2}\right)$ and 23$)\left(\min \chi_{t o t}^{2}\right)$ depend on one chosen parameter: $H_{0}, \Omega_{m 0}, \lambda_{m}, \lambda_{d}, \Omega_{k}$ and $w_{d}$. Here, for $\chi_{\Sigma}^{2}$ we compare two cases: for the model with 6 free parameters including $\Omega_{k} \neq 0$ where such dependencies are shown as blue thick lines, but for the flat case $\Omega_{k}=0$, the corresponding plots are black dashed lines. The graphs for $\chi_{\text {tot }}^{2}$ with CMB (red dash-dotted lines) are made for the general case $\Omega_{k} \neq 0$.

In particular, in the top-left panel of Fig. 1. the function $\min \chi_{\text {tot }}^{2}\left(H_{0}\right)$ means $\min _{\Omega_{m 0}, \Omega_{k}, w_{d}, \lambda_{m}, \lambda_{d}} \chi_{\text {tot }}^{2}$ (and the similar minimum for $\chi_{\Sigma}^{2}$ ). The $\chi^{2}$ absolute minima for these cases are presented in Table III with optimal values and $1 \sigma$ errors of model parameters. For each considered variant of the model (8) the corresponding line in Table III is obtained from the joint analysis $\mathrm{SNe}+H(z)+\mathrm{BAO}$ (for $\chi_{\Sigma}^{2}$ ) and the lower case of the line includes the absolute minimum of $\chi_{t o t}^{2}$ and estimations from the joint analysis $\mathrm{SNe}+H(z)+\mathrm{BAO}+\mathrm{CMB}$.

For example, for the variant $w_{d}=$ const with $\Omega_{k} \neq$ 0 , we estimate the Hubble constant $H_{0}=70.40_{-2.13}^{+2.18}$ $\mathrm{km}(\mathrm{s} \mathrm{Mpc})^{-1}$ for $\chi_{\Sigma}^{2}$ and $H_{0}=70.18_{-1.97}^{+1.77} \mathrm{~km}(\mathrm{~s} \mathrm{Mpc})^{-1}$ for $\chi_{\text {tot }}^{2} ; 1 \sigma$ errors are extracted from the one-dimensional likelihood function $\mathcal{L} \propto \exp \left(-\chi^{2} / 2\right)$.

The similar estimation for $\Omega_{m 0}$ is determined by the functions $\min \chi_{j}^{2}\left(\Omega_{m 0}\right)=\min _{H_{0}, \Omega_{k}, w_{d}, \lambda_{m}, \lambda_{d}} \chi_{j}^{2}$, if $\Omega_{k} \neq 0$; $j=\Sigma$, tot. These graphs for $\chi_{\Sigma}^{2}$ in the flat and non-flat cases are rather close and have distinct minimum with small $1 \sigma$ deviation $\Delta \Omega_{m 0} \simeq 0.013$. In the case $\chi_{t o t}^{2}$ the minimum is the same, but with smaller $\Delta \Omega_{m 0} \simeq 0.008$. It is connected with the factor $\sqrt{\Omega_{m 0}}$ in the values $A(z)$ in 30 and $R$ in 34 , so the contributions of $\chi_{B A O}^{2}$ and $\chi_{C M B}^{2}$ in the sum (23) are very sensitive to $\Omega_{m 0}$ values.

Dependence of min $\chi_{t o t}^{2}$ on the curvature $\Omega_{k}$ in the topright panel of Fig. 1 is strongly asymmetric, unlike the case of $\min \chi_{\Sigma}^{2}$. In these cases we have different $1 \sigma$ intervals for $\Omega_{k}$ (see Table III), but both include values $\Omega_{k} \simeq 0$. Some asymmetry may be seen for the plots $\min \chi^{2}\left(\lambda_{m}\right), \min \chi^{2}\left(\lambda_{d}\right)$ and $\min \chi^{2}\left(w_{d}\right)$ in the third row of panels. These calculations result in the estimations of $\lambda_{m}, \lambda_{d}$ and $w_{d}$ in Table III. 

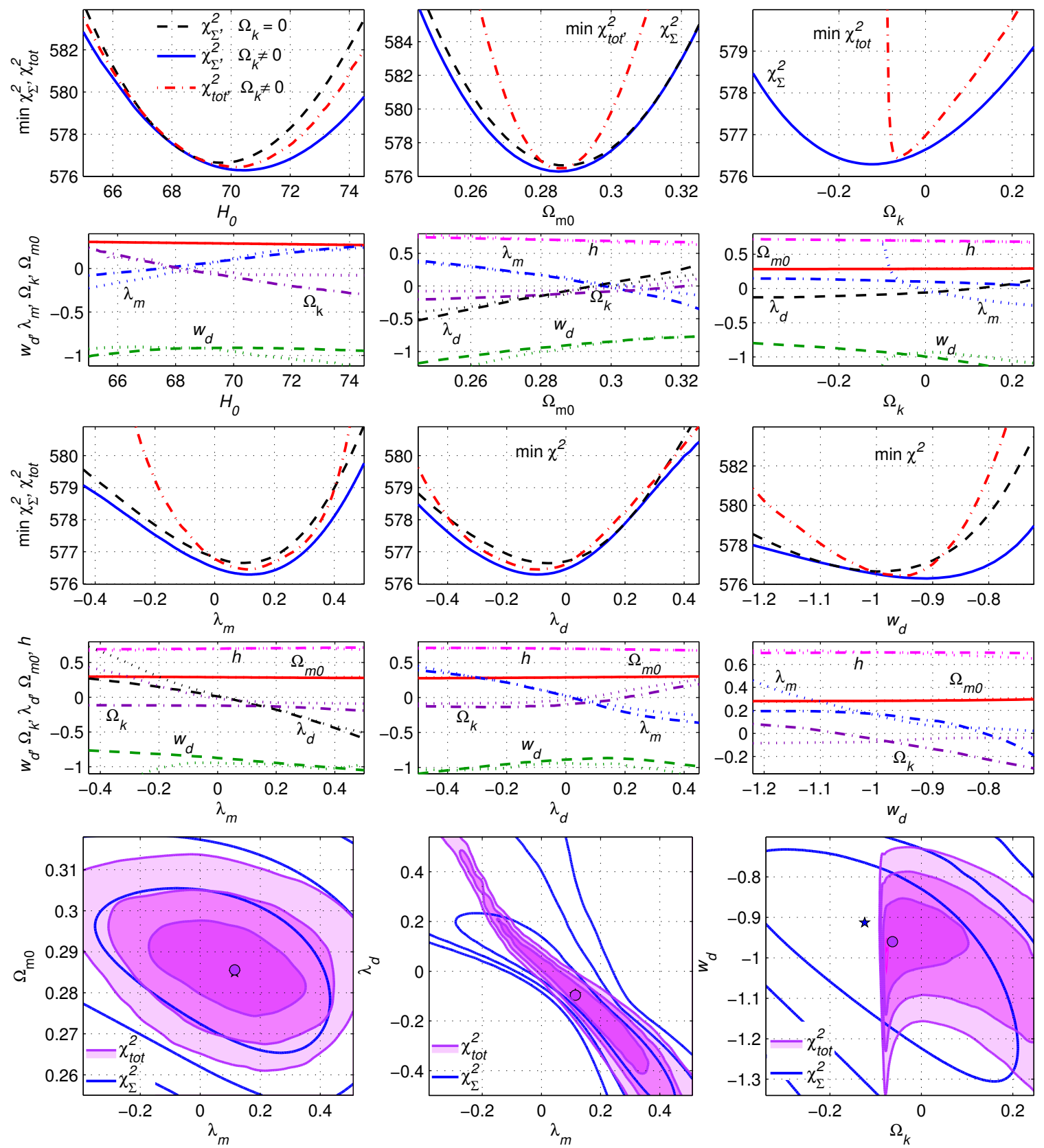

FIG. 1: For the model (8) with $w_{d}=$ const in the first and third rows of panels we present dependence of $\min \chi_{\Sigma}^{2}$ and $\min \chi_{\text {tot }}^{2}$ on $H_{0}, \Omega_{m 0}, \Omega_{k}, \lambda_{m}, \lambda_{d}$ and $w_{d}$ and also (in the panels below) the correspondent dependence for parameters of a minimum point. In the bottom panels the contour plots in the planes of 2 parameters are drawn at $1 \sigma, 2 \sigma$ and $3 \sigma$ confidence levels for $\chi_{\Sigma}^{2}$ (blue lines) and $\chi_{\text {tot }}^{2}$ (filled contours).

The panels in the second and forth rows of Fig. 1 correspond to the above panels and present dependencies of coordinates of minima points (optimal values of parameters) on $H_{0}, \ldots, \Omega_{k}$ for the function $\chi_{\Sigma}^{2}$ (if $\Omega_{k} \neq 0$ ) as thick lines and for $\chi_{\text {tot }}^{2}$ as dots. One can see that optimal values of $\lambda_{m}$ and $\lambda_{d}$ have distinct negative correlation (observed explicitly in the middle bottom panel), optimal values of $\Omega_{m 0}$ and $h=H_{0} / 100$ depend on other parameters rather weakly.
In 3 bottom panels of Fig. 1 we present the $1 \sigma$ (68.27\%), $2 \sigma(95.45 \%)$ and $3 \sigma(99.73 \%)$ contour plots for the functions $\min \chi_{j}^{2}\left(p_{1}, p_{2}\right)$ in the planes of two parameters. The minimum is calculated over the remaining 4 parameters. The mentioned level lines are shown for the $\chi_{\Sigma}^{2}$ function (blue curves) and for $\chi_{\text {tot }}^{2}$ as filled contours. The circles and stars in the plots mark minimum points obtained respectively for $\chi_{t o t}^{2}$ and $\chi_{\Sigma}^{2}$. 


\begin{tabular}{|c|c|c|c|c|c|c|c|c|}
\hline Variant & Data & $\min \chi^{2}$ & $H_{0}$ & $\Omega_{m 0}$ & $\lambda_{m}$ & $\lambda_{d}$ & $w_{d 0}$ & 6th parameter \\
\hline \multirow[t]{2}{*}{$w_{d}=$ const } & $\chi_{\Sigma}^{2}$ & 576.29 & $70.40_{-2.13}^{+2.18}$ & $0.285 \pm 0.013$ & $0.115_{-0.265}^{+0.217}$ & $-0.093_{-0.259}^{+0.230}$ & $-0.913_{-0.214}^{+0.132}$ & $\Omega_{k}=-0.124_{-0.190}^{+0.213}$ \\
\hline & $\chi_{t o t}^{2}$ & 576.45 & $70.18_{-1.97}^{+1.77}$ & $0.285 \pm 0.008$ & $0.115_{-0.193}^{+0.208}$ & $-0.097_{-0.240}^{+0.212}$ & $-0.955_{-0.113}^{+0.070}$ & $\Omega_{k}=-0.064_{-0.019}^{+0.102}$ \\
\hline \multirow{2}{*}{$\begin{array}{c}w_{d}=\text { const } \\
\& \Omega_{k}=0\end{array}$} & $\chi_{\Sigma}^{2}$ & 576.64 & $69.68_{-1.75}^{+1.80}$ & $0.287 \pm 0.013$ & $0.090_{-0.260}^{+0.213}$ & $-0.059_{-0.280}^{+0.233}$ & $-0.994_{-0.157}^{+0.123}$ & - \\
\hline & $\chi_{t o t}^{2}$ & 576.98 & $69.31_{-1.52}^{+1.67}$ & $0.285 \pm 0.008$ & $0.039_{-0.104}^{+0.193}$ & $-0.024_{-0.184}^{+0.168}$ & $-0.987_{-0.074}^{+0.096}$ & - \\
\hline \multirow{2}{*}{$\begin{array}{l}\text { Ansatz I } \\
(\alpha=0)\end{array}$} & $\chi_{\Sigma}^{2}$ & 576.29 & $69.55_{-1.73}^{+1.80}$ & $0.288 \pm 0.013$ & $0.173_{-0.32}^{+0.155}$ & $-0.277_{-0.309}^{+0.407}$ & $-0.94_{-0.157}^{+0.137}$ & $\beta=-0.25_{-0.54}^{+0.77}$ \\
\hline & $\chi_{t o t}^{2}$ & 576.93 & $69.86_{-1.68}^{+1.73}$ & $0.286 \pm 0.008$ & $0.245_{-0.34}^{+0.42}$ & $0.120_{-0.114}^{+0.136}$ & $-1.092_{-0.125}^{+0.148}$ & $\beta=0.34_{-0.475}^{+0.12}$ \\
\hline \multirow{2}{*}{$\begin{array}{l}\text { Ansatz II } \\
(\beta=0)\end{array}$} & $\chi_{\Sigma}^{2}$ & 576.57 & $69.66_{-1.75}^{+1.80}$ & $0.287 \pm 0.013$ & $0.123_{-0.175}^{+0.18}$ & $-0.112_{-0.26}^{+0.285}$ & $-0.988_{-0.135}^{+0.11}$ & $\alpha=0.073_{-0.055}^{+0.062}$ \\
\hline & $\chi_{\text {tot }}^{2}$ & 576.95 & $70.22_{-1.72}^{+1.69}$ & $0.288_{-0.008}^{+0.007}$ & $-0.035_{-0.08}^{+0.047}$ & $0.075_{-0.097}^{+0.125}$ & $-0.980_{-0.145}^{+0.13}$ & $\alpha=-0.032_{-0.064}^{+0.068}$ \\
\hline \multirow{2}{*}{$\begin{array}{l}\text { Ansatz III } \\
(\alpha=1)\end{array}$} & $\chi_{\Sigma}^{2}$ & 576.64 & $69.68_{-1.74}^{+1.80}$ & $0.287 \pm 0.013$ & $0.098_{-0.24}^{+0.475}$ & $-0.060_{-0.266}^{+0.74}$ & $-0.996_{-0.155}^{+0.12}$ & $\beta=0.997_{-0.115}^{+0.243}$ \\
\hline & $\chi_{t o t}^{2}$ & 577.33 & $68.82_{-1.35}^{+1.48}$ & $0.290 \pm 0.007$ & $0.022_{-0.048}^{+0.093}$ & $-0.548_{-0.42}^{+0.58}$ & $\begin{array}{l}-0.96_{-0.142}^{+0.095} \\
\end{array}$ & $\beta=0.235_{-0.10}^{+0.216}$ \\
\hline \multirow{2}{*}{$\begin{array}{l}\text { Ansatz IV } \\
\text { Eq. } 20\end{array}$} & $\chi_{\Sigma}^{2}$ & 576.07 & $69.23_{-1.86}^{+1.90}$ & $0.292_{-0.015}^{+0.016}$ & $0.237_{-0.250}^{+0.076}$ & $-0.452_{-0.43}^{+0.73}$ & $-0.786_{-0.31}^{+0.356}$ & $w_{1}=-3.14_{-4.72}^{+4.30}$ \\
\hline & $\chi_{\text {tot }}^{2}$ & 576.90 & $69.14_{-1.62}^{+1.74}$ & $0.285 \pm 0.008$ & $0.016_{-0.038}^{+0.044}$ & $-0.054_{-0.110}^{+0.096}$ & $-0.925_{-0.215}^{+0.23}$ & $w_{1}=-0.68_{-1.14}^{+0.90}$ \\
\hline \multirow{2}{*}{$\begin{array}{l}\text { Ansatz V } \\
\text { Eq. } 21\end{array}$} & $\chi_{\Sigma}^{2}$ & 575.97 & $69.32_{-1.74}^{+1.84}$ & $0.292 \pm 0.015$ & $0.220_{-0.245}^{+0.062}$ & $-0.497_{-0.386}^{+0.64}$ & $-0.810_{-0.27}^{+0.338}$ & $w_{1}=-2.68_{-4.03}^{+3.75}$ \\
\hline & $\chi_{\text {tot }}^{2}$ & 576.92 & $69.18_{-1.65}^{+1.70}$ & $0.292 \pm 0.008$ & $0.023_{-0.042}^{+0.030}$ & $-0.092_{-0.120}^{+0.084}$ & $-0.822_{-0.255}^{+0.192}$ & $w_{1}=-0.43_{-1.57}^{+0.65}$ \\
\hline
\end{tabular}

TABLE III: Variants of the model (8) and $1 \sigma$ estimates of the model parameters using the joint analysis $\mathrm{SNe}+H(z)+\mathrm{BAO}($ the upper case on all lines) and $\mathrm{SNe}+H(z)+\mathrm{BAO}+\mathrm{CMB}$ (the lower case on all lines).

\subsection{Results for $w_{d} \neq$ constant.}

For the variable EoS in DE, presented in section 2.2. we summarize their observational constraints in Table III for both $\mathrm{SNe}+H(z)+\mathrm{BAO}$ and $\mathrm{SNe}+H(z)+\mathrm{BAO}+\mathrm{CMB}$. We consider several possibilities for variable $w_{d}$. The first one is very general given in Eq. (14) and it provides three distinct possibilities in equations (16), (17) and (18) while additionally we consider CPL (20) and linear parametrizations (21). The general ansatz (14) with its solution (15) gives not only new possibilities, but also additional problems of the following two types: (i) two extra model parameters $\left(3\right.$ parameters $w_{d 0}, \alpha$ and $\beta$ instead of one $w_{d}$ ); (ii) singularities in the past, which appear in different scenarios of the class 15 .

These singularities connected with bad behavior of densities $\rho_{d m}, \rho_{d}$ or their sum $\rho_{T}$ at a moment $t_{s}$ in the past, when the scale factor remains finite and nonzero $\left(a\left(t_{s}\right) \neq 0\right)$, they may be classified into the following three types:

$$
\begin{array}{ll}
\text { a) } & \lim _{t \rightarrow t_{s}} \rho_{T}=\infty ; \\
\text { b) } & \rho_{d m}<0, \text { if } t<t_{s} ; \\
\text { c) } & \rho_{d}<0, \text { if } t<t_{s} .
\end{array}
$$

These cases resemble classification of singularities in Refs. 91 93. For singularities (35) of the type (c) DE pressure $p_{d}\left(t_{s}\right)$ remains finite at the moment $t_{s}$, whereas $\rho_{d}\left(t_{s}\right)=0$; they may be also divided into class (c1) with $p_{d}\left(t_{s}\right)=0$ and finite $w_{d}=p_{d} / \rho_{d}$ and class (c2) with $p_{d}\left(t_{s}\right) \neq 0$, where $w_{d}$ tends to infinity if $t \rightarrow t_{s}$. Possible singularities (35) compel us to be especially careful, when we calculate numerically parameters of effective scenarios in this model. We should exclude domains in parameter
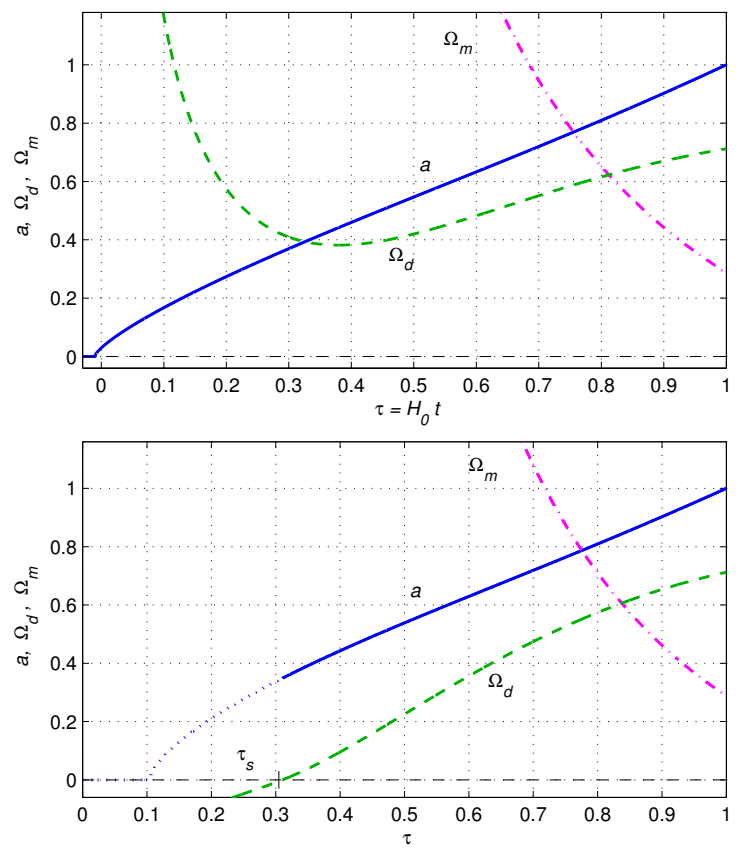

FIG. 2: Evolution of the scale factor $a(\tau)$ and densities $\Omega_{m}(\tau), \Omega_{d}(\tau)$ for Ansatz I 16 is shown for the regular solution (top) with optimal parameters from Table III and for the singular solution (bottom) with type (c1) singularity (35) (here $\lambda_{m}=-0.01$, other parameters are the same).

space with singular behavior of physical densities irrespective of type (35). The example of singular solution with the type (c1) singularity 35 is shown in Fig. 2

In Fig. 2 we compare the regular solution for Ansatz I 16. (the top panel) with the singular solution in the bottom panel. One can see how the scale factor $a$ (blue solid 
lines) and densities $\Omega_{m}=\rho_{m} / \rho_{c r}$ (magenta dash-dotted lines) and $\Omega_{d}(\tau)=\rho_{d} / \rho_{c r}$ (green dashed lines) depend on dimensionless time $\tau=H_{0} t$. Here $\rho_{c r}=3 H_{0}^{2} / 8 \pi G$ is the critical density of the universe. The model parameters are taken with their optimal values from Table III for $\chi_{\Sigma}^{2}$, but for the type (c1) singularity (the bottom panel) with difference only in one value: $\lambda_{m}=-0.01$. In this singular case the DE density $\Omega_{d}$ becomes negative at $\tau<\tau_{s}$.

We mentioned above, that the number $N_{p}$ of model parameters for scenarios with variable $w_{d}$ satisfying Eq. (14) is too large, it is disadvantage in competition with other models in accordance with information criteria [94 96. So we have to exclude non-flat scenarios and fix in this section $\Omega_{k}=0$. But even for the flat case we have $N_{p}=7$ parameters: $H_{0}, \Omega_{m 0}, \lambda_{m}, \lambda_{d}, w_{d 0}, \alpha, \beta$.

An attempt to exclude $\lambda_{m}$ appeared to be unsuccessful: though in the case $\lambda_{m}=0$, we can avoid some singularities (35) and instabilities in perturbations 97, 98, but the best value of the function 23 min $\chi_{\Sigma}^{2} \simeq 576.74$ is worth, than in the case $w_{d}=$ const 10 (but $\lambda_{m} \neq 0$ ). So we have to fix other parameters. First, we consider the case $\alpha=0(16)$, denoted in Sect. 2.2 as Ansatz I.

For Ansatz I $(\alpha=0)$ we have no acceptable analytic solution of Eq. (9), so we investigate numerical solutions of the system (11), (3), (4), (8), (16) with natural initial conditions $\left.\rho_{m}\right|_{t=t_{0}}=\rho_{m 0},\left.\rho_{d}\right|_{t=t_{0}}=\rho_{d 0}$ at the present day and integration "into the past". For Ansatz I we can reach the best values $\min \chi_{\Sigma}^{2} \simeq 576.29$ (this solution is shown in Fig. 2 in the top panel) and $\min \chi_{\text {tot }}^{2} \simeq 576.93$. The corresponding values of model parameters are tabulated in the "Ansatz I" line of Table 【II.

We analyze the flat case of Ansatz I (16) in Fig. 3. For one dimensional distributions and contour plots we use notations of Fig. 11 the red dashed lines and filled contours for $\min \chi_{t o t}^{2}$ and the blue lines for $\min \chi_{\Sigma}^{2}$. In 3 panels of Fig. 3 (upper left; upper middle and lower left) we compare this variant of the model with Ansatz II (17) (the green lines). One should note that for both cases the optimal values of parameters (in particular, for $\lambda_{m}, \lambda_{d}$ ) do not coincide for $\chi_{t o t}^{2}$ and $\chi_{\Sigma}^{2}$. In other words, when we include the CMB contribution $\chi_{C M B}^{2}(34)$ into the function $\chi_{t o t}^{2}=\chi_{\Sigma}^{2}+\chi_{C M B}^{2}$, the resulting minimum point for $\chi_{t o t}^{2}$ appears to be shifted. This effect can be seen in the bottom-right panel of Fig. 3, where for the contour plots of Ansatz I the circle and star respectively mark the minimum points for $\chi_{\text {tot }}^{2}$ and $\chi_{\Sigma}^{2}$ (see also the one dimensional distributions for $\min \chi^{2}\left(\lambda_{d}\right)$ and $\left.\min \chi^{2}(\beta)\right)$.

As a consequence of this behavior we have the absolute minima of $\chi_{\text {tot }}^{2}$ for Ansatz I and Ansatz II in Table III only a bit better than the value 576.98 for the case $w_{d}=$ const with $\Omega_{k}=0$. Note that the both variants turn into this case, if we take $\beta=0$ and $\alpha=0$ in Eqs. (16), (17), respectively.

When we compare the variants of the model (14), we keep in mind that Ansatz I $(\alpha=0)$ and Ansatz II (17) $(\beta=0)$ have the same number of model parameters $N_{p}=$ 6 , but for the case of $w_{d}=$ const with $\Omega_{k}=0$, this value is $N_{p}=5$. We try to minimize $N_{p}$, hence, here and below for all variants of the model, we consider only the flat case $\left(\Omega_{k}=0\right)$. In Fig. 3 we compare both Ansatz I and Ansatz II where we see that for Ansatz I, the essential advantage is in the absolute minimum of $\chi_{\Sigma}^{2}$, but small for $\chi_{t o t}^{2}$.

Dependence of $\min \chi_{\Sigma}^{2}$ and $\min \chi_{\text {tot }}^{2}$ on $\Omega_{m 0}$ in the bottom-left panel of Fig. 3 is similar for both presented variants of the model, but the upper curves for $\chi_{t o t}^{2}$ are more narrow. As usual, these minima are taken over all remaining parameters. In other panels we see different dependence of these minima on $\lambda_{m}, \lambda_{d}$ and $\beta$. These behavior in some cases are connected with various singularities (35), which can appear in certain domains of the parameter space.

In Fig. 4 we demonstrate how the most successful variants of the model with parameters from Table III describes SNe data [59] with the functions $D_{L}(z)$ (the upper panel) and $H(z)$ data from TableI (the middle panel); in the bottom panel we draw the corresponding plots of the interaction function (8): $Q(z)=3 H\left(\lambda_{m} \rho_{d m}+\lambda_{d} \rho_{d}\right)$ in its dimensionless form $Q(z) / Q_{0}$, where $Q_{0}=H_{0} \rho_{c r}=$ $3 H_{0}^{3} /(8 \pi G)$. The $H(z)$ data in the middle panel are marked as cyan or magenta stars, if they are obtained from differential ages or from BAO data. From Fig. 4 , we see that the plots of $D_{L}(z)$ and $H(z)$ are practically coincide for all considered variants.

For the five most successful variants of the model, the dotted lines correspond to the optimal parameters for $\chi_{\text {tot }}^{2}(\mathrm{SNe}+H(z)+\mathrm{BAO}+\mathrm{CMB})$; the solid and dashed lines describe the minimization of $\chi_{\Sigma}^{2}$ $(\mathrm{SNe}+H(z)+\mathrm{BAO})$. We observe that the observational data $\mathrm{SNe}+H(z)+\mathrm{BAO}$ always suggest that there is a transition of $Q$ at late time from its positive values to negative values, and the transion occurs around $z \simeq$ 0.4. On the other hand, for the observational data $\mathrm{SNe}+H(z)+\mathrm{BAO}+\mathrm{CMB}$, except for Ansatz I (in this case $Q$ remains positive throughout the evolution of the universe), all other variants keep the same behaviour as we observe for the data $\mathrm{SNe}+H(z)+\mathrm{BAO}$. That means $Q$ changes its sign from positive to negative values around the same redshift. Thus, we find that almost all variants allow the flow of energy from CDM to DE at late time (precisely for $z \lesssim 0.4$ ) while at for $z \gtrsim 0.4$, the energy flow takes place from DE to CDM. Moreover, for $w_{d}=$ constant (both for $\Omega_{k}=0$ and $\Omega_{k} \neq 0$ ), as seen from the Fig. 4. the quantity $Q / Q_{0}$ is very very close to zero, that means, a very small interaction is favored in this case. It is an interesting result becasue some other interactions also conclude very small interaction in the dark sector for constant $w_{d}$, see [30, 31, 36.

For the Ansatz III (18) the best values of $\min \chi_{\Sigma}^{2}$ and $\min \chi_{\text {tot }}^{2}$ (see Table III) are worse, than the corresponding minima for other variants of the model with the same $N_{p}=6$. The main drawback of Ansatz III is that its solutions behave badly with the optimal parameters: they are close to singular solutions of types (a) and (c) 35 . So in our calculations of values in Table III we had to bypass singular domains in the parameter space. We in- 

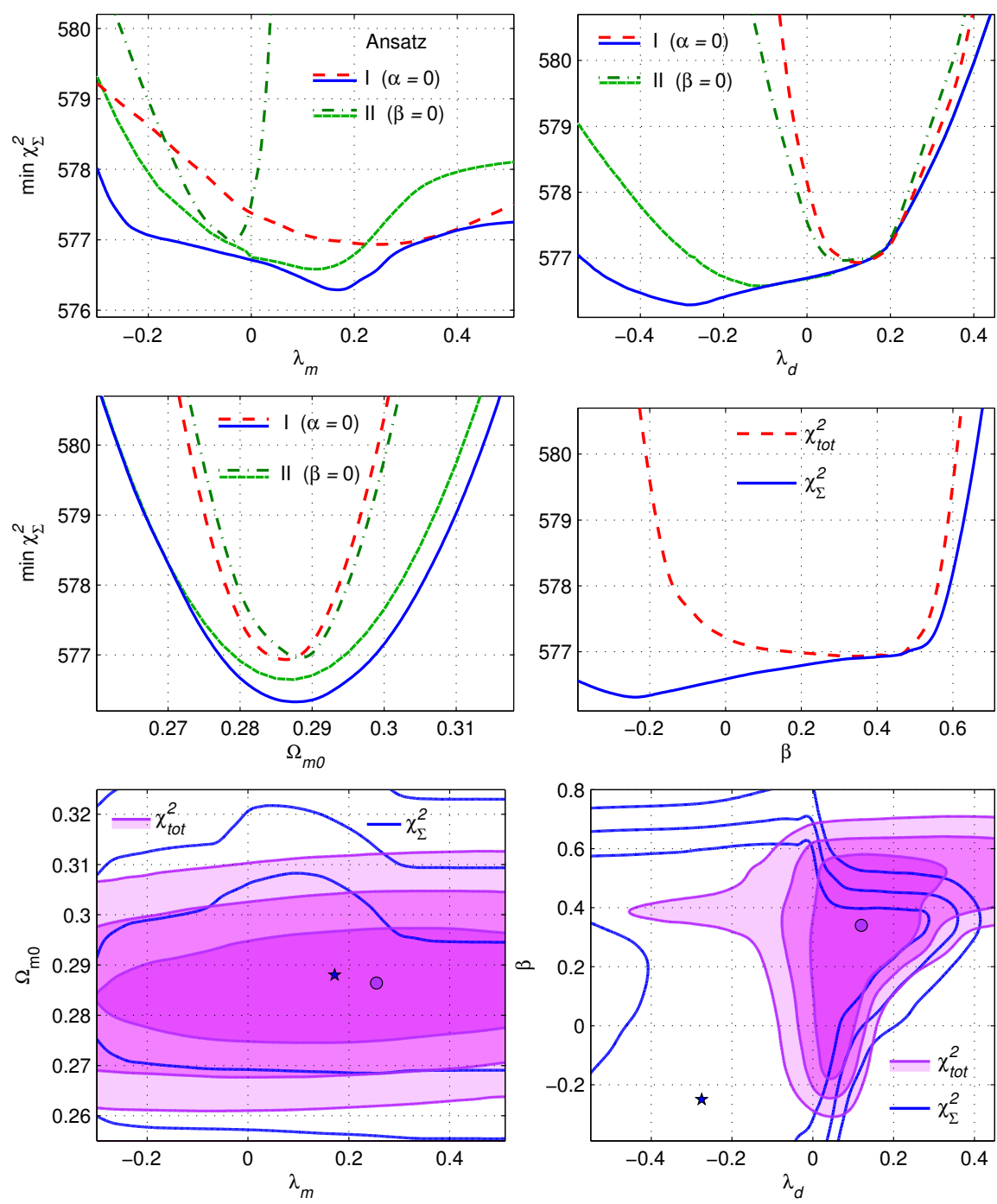

FIG. 3: For Ansatz I 16 and Ansatz II 17 with $\Omega_{k}=0$ we present one dimensional distributions of $\min \chi_{\Sigma}^{2}$ and $\min \chi_{\text {tot }}^{2}$. For Ansatz I we also draw two dimensional contour plots with notations from Fig. 1. the blue lines for $\chi_{\Sigma}^{2}$, but the filled contours and red dashed lines for $\chi_{t o t}^{2}$. We note that the circles and stars in the plots mark minimum points obtained respectively for $\chi_{\text {tot }}^{2}$ and $\chi_{\Sigma}^{2}$.

vestigate the same ansatz (Ansatz III) with the choice $\lambda_{m}=0$, considered in Ref. [49 since the background is analytically solved for this case, see eq. $(19)$. For this model our analysis shows that $\min \chi_{\Sigma}^{2} \simeq 576.81$ and $\min \chi_{\text {tot }}^{2} \simeq 577.46$. Thus, it is seen that this variant with analytic solutions appeared to be unsuccessful in comparison with the case (10): $w_{d}=$ const, $\Omega_{k}=0$ (for these variants $N_{p}=5$, so they are comparable). Due to these reasons we do not present the constraints for $\lambda_{m}=0$, $\alpha=1$ in a separate line in Table III. Thus, we observe that Ansatz III for both choices $\lambda_{m} \neq 0$ and $\lambda_{m}=0$ is not suitable as reported by the observational data. So, we do not present any graphical analysis for this ansatz.

The next variant 20) (Ansatz IV) behaves better for $\chi_{\Sigma}^{2}$. It has only the type (c1) singularities in the domain $\lambda_{m}<0$. This domain is far from optimal values of the model parameters for $\chi_{\Sigma}^{2}$ with the smallest $\min \chi_{\Sigma}^{2} \simeq$ 576.07 (see Table III). However, the minimum of $\chi_{\text {tot }}^{2}$ is achieved in the $\lambda_{m}<0$ domain. So, the minimal value 576.88 of $\chi_{\text {tot }}^{2}=\chi_{\Sigma}^{2}+\chi_{C M B}^{2}$ appears to be rather large. This behavior is seen in the top-left panel of Fig. 5. where the red dash-dotted line for $\chi_{\Sigma}^{2}$ and the dashed magenta line for $\chi_{t o t}^{2}$ show, how the minima $\min _{\Omega_{m 0}, \lambda_{d}, H_{0}, w_{d 0}, w_{1}} \chi^{2}$ depend on $\lambda_{m}$.

For the last variant (21) (Ansatz V), we achieve the absolute minimum for $\chi_{\Sigma}^{2}$ among all considered models in Tables III and IV $\min \chi_{\Sigma}^{2} \simeq 575.97$. However, if we add the CMB, minimum of $\chi_{t o t}^{2}$ becomes rather large, because it is achieved near the $\lambda_{m}<0$ domain. In this domain solutions have the type (c) singularity (35), so it is practically forbidden.

In Fig. 5 one can see the one dimensional distributions 

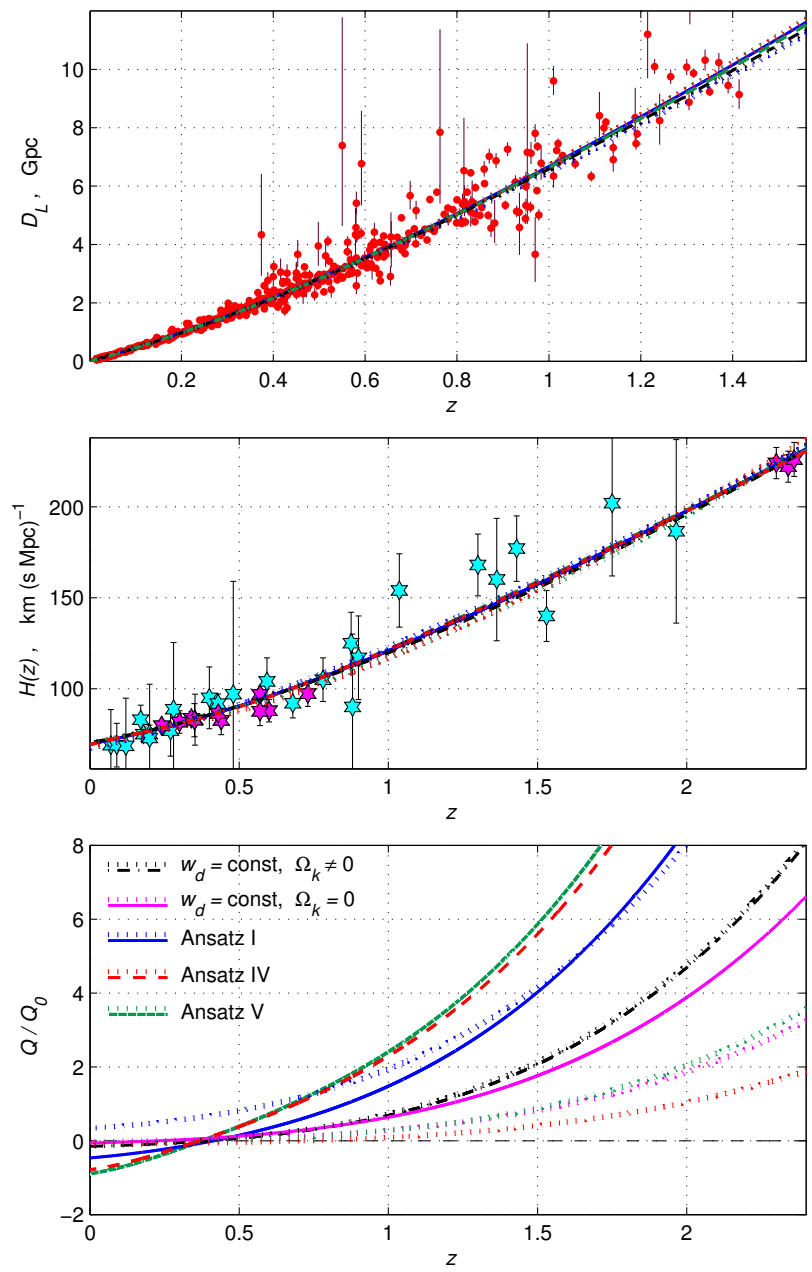

FIG. 4: For different variants of the model with optimal parameters from Table III we show the plots for $D_{L}(z)$ (upper panel) describing the SNe data 59, $H(z)$ functions with the data from Table I (middle panel) and $Q(z)$ dependence (lower panel). We note that the same labels in the lower panel follow for the other two plots (i.e. upper and middle plots). We further note that the plots for different variants of the models both in upper and middle panel are almost indistingushable from each other while although in the lower panel the plots for $Q(z) / Q_{0}$ are distingushable for large reshift but for low redshifts they are also indistinguishable from each other.

of $\min \chi_{\Sigma}^{2}$ and $\min \chi_{\text {tot }}^{2}$ for Ansatz IV (20) and Ansatz $\mathrm{V}$ (21). In the bottom panels we draw two dimensional contour plots for Ansatz IV with filled contours for $\chi_{\text {tot }}^{2}$ and red lines for $\chi_{\Sigma}^{2}$. Here, we use notations from Figs. 1 and 3 . In particular, the circles and stars demonstrate difference between minimum points for $\chi_{\text {tot }}^{2}$ and $\chi_{\Sigma}^{2}$. A striking feature that one must note is in the behaviour of the $w_{1}$ parameter in Ansatz IV 20) and Ansatv V (21). From Table III, one can see that the value of $w_{1}$ for both ansatze (Ansatz IV and Ansatz V) significantly chnages after the inclusion of the CMB data.

\section{INTERACTING AND NON-INTERACTING MODELS: A STATISTICAL COMPARISON}

In this section we compare our interacting dark energy scenario with some other existing non-interacting cosmological models purely from the statistical ground. In Table IV we demonstrate how these models describe the same observational data for SNe Ia [59], $H(z)$ and BAO from Tables I. II. Calculations were made in accordance with the procedure described in Sect. 3. and in Ref. 90. Therefore, we will briefly describe the models in the following subsections.

\subsection{Modified Chaplygin gas and its family}

The equation of state for modified Chaplygin gas (MCG) with pressure $p_{g}$ and energy density $\rho_{g}$ is 99,100

$$
p_{g}=A \rho_{g}-\frac{B}{\rho_{g}^{\alpha}} .
$$

Modified Chaplygin gas is the subsequent generalizations of Chaplygin gas (EoS: $p_{g}=-B / \rho_{g}$ ) and generalized Chaplygin gas (GCG) with EoS [101]:

$$
p_{g}=-B / \rho_{g}^{\alpha} .
$$

In these models GCG or MCG acts as a unified candidate for dark matter and dark energy.

In Table IV for the MCG and GCG models we cite the results of calculations from Ref. [90, where these scenarios were explored as two-component models with usual dust-like baryonic matter component $\rho_{b}$ and the Chaplygin gas component $\rho_{g}: \rho=\rho_{b}+\rho_{g}$. In this case the Friedmann equation (1) is

$$
\begin{aligned}
& H^{2} / H_{0}^{2}=\Omega_{b 0} a^{-3}+\Omega_{k} a^{-2} \\
+ & \left(1-\Omega_{b 0}-\Omega_{k}\right)\left[B_{s}+\left(1-B_{s}\right) a^{-3(1+A)(1+\alpha)}\right]^{1 /(1+\alpha)} .
\end{aligned}
$$

Here the dimensionless parameter $B_{s}=B \rho_{g 0}^{-1-\alpha} /(1+A)$ is used instead of $B$, and $\rho_{g 0}=\rho_{g}\left(t_{0}\right)$.

For MCG, GCG and other cosmological models the estimations in Table IV were made for the value $\Omega_{b 0}=$ 0.044 (Eq. (32)). It is shown in Ref. 90, that this value is optimal for the $\Lambda \mathrm{CDM}, \mathrm{MCG}, \mathrm{GCG}$ and the model with $\operatorname{EoS}$ (38), if we use the fitting formula (31). These estimations were supported with the more simple fitting formula $r_{d}=\left(r_{d} h\right)_{f i d} \cdot h^{-1}$, but in the latter case the mentioned models are not sensitive to a value $\Omega_{b 0}$ in the range $0 \leq \Omega_{b 0} \leq 0.15$, because of similarity in properties of dark matter and baryonic matter. Due to this reason we do not consider $\Omega_{b 0}$ as a free model parameter and fix it in the form 32 for all models in Table IV

One can see in Table IV that the MCG model demonstrates the value $\min \chi_{\Sigma}^{2}=576.45$, it is a bit better than the the $w_{d}=$ const model $10 p$. The MCG mode also has $N_{p}=5$ parameters: $H_{0}, \Omega_{k}, A, B_{s}, \alpha$. For the GCG 

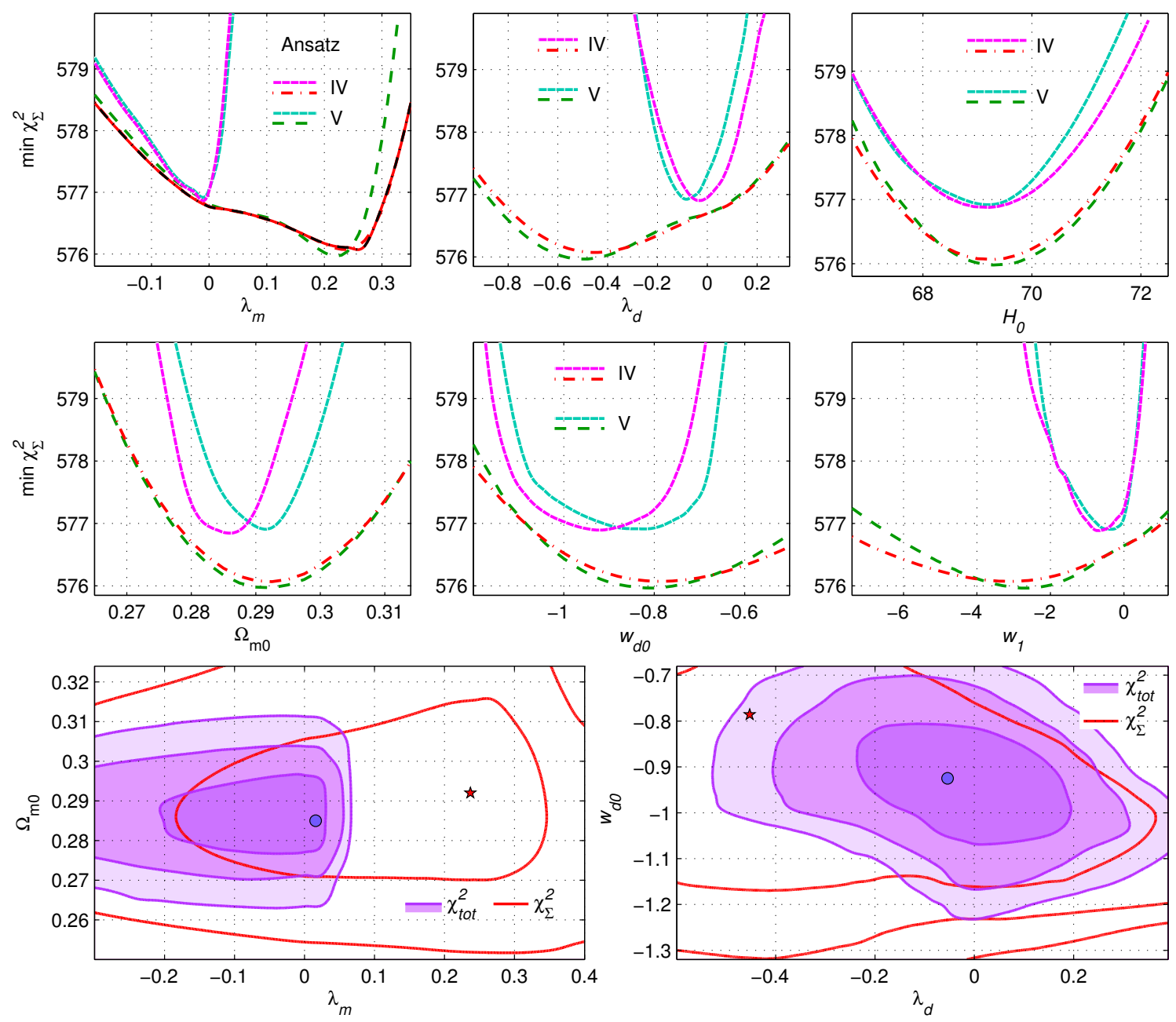

FIG. 5: Dependence of $\min \chi_{\Sigma}^{2}$ on $H_{0}, \lambda_{m}, \lambda_{d}, \Omega_{m 0}, w_{d 0}, w_{1}$ for Ansatz IV 20 (red and magenta lines) and for Ansatz V 21) (green and aquamarine lines). The contour plots with $1 \sigma, 2 \sigma$ and $3 \sigma$ confidence levels in the bottom panels are shown for Ansatz IV in notations of Fig. 1 where the red lines stands for $\chi_{\Sigma}^{2}$ and the filled one for $\chi_{\text {tot }}^{2}$. The circles and stars in the contour plots mark minimum points obtained respectively for $\chi_{t o t}^{2}$ and $\chi_{\Sigma}^{2}$.

model the minimum of $\chi_{\Sigma}^{2}$ is worse, however in this case we have $N_{p}=4$ parameters (because $A=0$ ), so the GCG model gets advantage from information criteria.

\subsection{Quadratic equation of state}

We consider a cosmic substratum having quadratic equation of state which has similar unified behavior as in MCG. Further, this quadratic EoS asymptotically becomes of de Sitter type. The EoS [90, 102]

$$
p=\tilde{p}_{0}+w_{0} \rho_{g}+\tilde{\beta} \rho_{g}^{2}
$$

includes the first three terms of the Taylor series expansion of an arbitrary function $p=f\left(\rho_{g}\right)$, where $\tilde{p}_{0}, w_{0}, \tilde{\beta}$ are free parameters. It is convenient to rewrite this EoS in the form 90.

$$
p=p_{0} \rho_{c r}+w_{0} \rho_{g}+\beta \rho_{g}^{2} / \rho_{c r},
$$

where $p_{0}=\tilde{p}_{0} / \rho_{c r}, \beta=\tilde{\beta} \rho_{c r}$ are the dimensionless parameters and $\rho_{c r}=3 H_{0}^{2} / 8 \pi G$.

Solving the conservation equation (2), we obtain the energy density $\rho_{g}$ in the form

$$
\frac{\rho_{g}}{\rho_{c}}= \begin{cases}\frac{1}{2 \beta}\left[\frac{\Gamma-\sqrt{|\Delta|} \tan \left(\frac{x}{2} \sqrt{|\Delta|}\right)}{1-|\Delta|^{-\frac{1}{2}} \tan \left(\frac{x}{2} \sqrt{|\Delta|}\right)}-1-w_{0}\right], & \Delta<0, \\ \frac{1}{2 \beta}\left[\left(\frac{x}{2}+\frac{1}{\Gamma}\right)^{-1}-1-w_{0}\right], & \Delta=0, \\ \frac{\rho-\left(\Omega_{m}-\rho_{+}\right) a^{-3 \sqrt{\Delta}}-\rho_{+}\left(\Omega_{m}-\rho_{-}\right)}{\left(\Omega_{m}-\rho_{+}\right) a^{-3 \sqrt{\Delta}}-\Omega_{m}+\rho_{-}}, & \Delta>0\end{cases}
$$

where $\Delta=\left(1+w_{0}\right)^{2}-4 \beta p_{0}, \Omega_{m}=1-\Omega_{k}-\Omega_{b 0}, \Gamma=$ $2 \beta \Omega_{m}+1+w_{0}, \rho_{ \pm}=\frac{-1-w_{0} \pm \sqrt{\Delta}}{2 \beta}$.

Hence, the evolution equation can be written as

$$
H^{2}=H_{0}^{2}\left[\frac{\rho_{g}}{\rho_{c}}+\Omega_{b 0} a^{-3}+\Omega_{k} a^{-2}\right] .
$$

The value $\min \chi_{\Sigma}^{2}=576.03$ for the model $(38)$ in Table IV is better than for the MCG model, it is close to 


\begin{tabular}{l|c|c|c|c|c|c|c|c}
\hline Model & $\min \chi_{\Sigma}^{2}$ & $\min \chi_{\text {tot }}^{2}$ & $\frac{\min \chi_{\text {tot }}^{2}}{\text { d.o.f }}$ & $N_{p}$ & $A I C_{\Sigma}$ & $A I C_{\text {tot }}$ & $\Delta A I C_{\text {tot }}$ & $\Delta B I C_{\text {tot }}$ \\
\hline$w_{d}=$ const (non-flat) & 576.29 & 576.45 & 0.9107 & 6 & 588.29 & 588.45 & 2.46 & 15.84 \\
\hline$w_{d}=$ const (flat) & 576.44 & 576.98 & 0.9101 & 5 & 586.44 & 586.98 & 0.99 & 9.91 \\
\hline Ansatz I [Eq. 16]] & 576.29 & 576.93 & 0.9114 & 6 & 588.29 & 588.93 & 2.94 & 16.32 \\
\hline Ansatz IV [Eq. [20]] & 576.07 & 576.90 & 0.9114 & 6 & 588.07 & 588.90 & 2.91 & 16.29 \\
\hline Ansatz V [Eq. [21]] & 575.97 & 576.92 & 0.9114 & 6 & 587.97 & 588.92 & 2.93 & 16.31 \\
\hline$\Lambda$ CDM & 578.56 & 579.99 & 0.9119 & 3 & 584.56 & 585.99 & 0 & 0 \\
\hline GCG [Eq. [37)] & 577.01 & 578.26 & 0.9106 & 4 & 585.01 & 586.26 & 0.27 & 4.73 \\
\hline MCG [Eq. [36]] & 576.45 & 577.62 & 0.9111 & 5 & 586.45 & 587.62 & 1.63 & 10.55 \\
\hline Quadratic [Eq. [38]] & 576.03 & 577.46 & 0.9108 & 5 & 586.03 & 587.46 & 1.47 & 10.39 \\
\hline CPL [Eq. 39]] & 576.57 & 577.83 & 0.9114 & 5 & 586.57 & 587.83 & 1.84 & 10.76 \\
\hline Linear [Eq. [40]] & 576.57 & 577.74 & 0.9113 & 5 & 586.57 & 587.74 & 1.75 & 10.67 \\
\hline
\end{tabular}

TABLE IV: A statistical comparison of some successful variants of the interacting dark energy model with some well known non-interacting cosmological models has been presented using two different combined analysis $\mathrm{SNe}+H(z)+\mathrm{BAO}$ and $\mathrm{SNe}+H(z)+\mathrm{BAO}+\mathrm{CMB}$.

the best result of Ansatz V (21).

\subsection{CPL parametrization}

We also consider the universe including cold dark matter and a dark energy component with ChevallierPolarski-Linder (CPL) parametrization [53, 54, i.e. Eq. 20.

$$
w=w_{0}+w_{1} \frac{z}{1+z}=w_{0}+w_{1}(1-a)
$$

where $w_{0}, w_{1}$ are two free parameters. In presence of this dark energy component (with its present time fraction $\left.\Omega_{X 0}=1-\Omega_{m 0}-\Omega_{k}\right)$ the evolution equation is

$$
\frac{H^{2}}{H_{0}^{2}}=\Omega_{m 0} a^{-3}+\Omega_{k} a^{-2}+\Omega_{X 0} a^{-3\left(1+w_{0}+w_{1}\right)} e^{3 w_{1}(a-1)} .
$$

It is interesting to compare this model with the considered above variant (20) (Ansatz IV) of our interacting model with the similar EoS. In other words, the CPL model (39) transforms into Ansatz IV (20), if we include the interaction term (8) with two model parameters $\lambda_{m}$, $\lambda_{d}$ and fix the curvature parameter $\Omega_{k}=0$.

From Table [V] we see that the interacting scenario 20. (Ansatz IV) has the essential advantage $\left(\min \chi_{\Sigma}^{2} \simeq\right.$ 576.07 and $\left.\min \chi_{\text {tot }}^{2} \simeq 576.9\right)$ in compared to the noninteracting model $(39)\left(\min \chi_{\Sigma}^{2} \simeq 576.57, \min \chi_{t o t}^{2} \simeq\right.$ 577.83).

\subsection{Linear parametrization}

The model with linear parametrization in EoS is similar to the considered above CPL parametrization (39), but has the following EoS [56 58.

$$
w=w_{0}+w_{1} z
$$

where $w_{0}, w_{1}$ are two free parameters to be constrained by the observational data. The evolution equation for a universe made of cold matter and the dark energy with the above equation of state is

$$
\frac{H^{2}}{H_{0}^{2}}=\Omega_{m 0} a^{-3}+\Omega_{k} a^{-2}+\Omega_{X 0} a^{-3\left(1+w_{0}-w_{1}\right)} e^{3 w_{1}\left(\frac{1-a}{a}\right)} .
$$

One can see in Table IV] and in Fig. 5 that the model 40 behaves very closely to the CPL scenario (39), but it demonstrates essentially worse $\min \chi_{\Sigma}^{2}$ than the corresponding interactive model 21) (Ansatz V).

Hierarchy of the scenarios in Table [V] will change, if we take into account information criteria which use a number $N_{p}$ of model parameters (degrees of freedom). In particular, the Akaike and Bayesian information criteria are given by 94,96 .

$$
A I C=\min \chi_{\Sigma}^{2}+2 N_{p}, \quad A I C=\min \chi_{\Sigma}^{2}+N_{p} \log N,
$$

where $N$ is the number of data points used in the fit. These criteria give advantage to the $\Lambda \mathrm{CDM}$ and other models with minimal $N_{p}$.

\section{SUMMARY}

In the FLRW background of our Universe we have considered an interacting scenario between dark matter and dark energy where both of them obey barotropic equation of state. The interaction is a linear combination of the energy densities of the dark components in the form $Q=3 H \lambda_{m} \rho_{m}+3 H \lambda_{d} \rho_{d}$, where $\left(\lambda_{m}, \lambda_{d}\right)$ are the coupling parameters describing the strength and direction of energy flow from their sign (i.e. whether $Q>0$ or $Q<0)$. Since the EoS in DE could be either constant or variable, hence we have examined both the possibilities to explore the cosmological scenarios with the use of 
current astronomical data. For $w_{d}=$ constant, the evolution equations for matter and dark energy take analytic forms. For variable $w_{d}$ we have proposed three ansatze in Eqns. (16), 17), (18), which emerge from the generalized ansatz given in Eq. (14). In addition to these, we have considered two more variable EoS in DE in the forms of CPL and linear parametrizations in equations 20, 21, , respectively. Altogether, we have considered 7 variants for the present interacting model for a detailed analysis.

Henceforth, with the introduction of 7 variants of the EoS in dark energy, we constrained the model parameters using the joint analysis of Union 2.1, Hubble parameter measurements, baryon acoustic oscillation data points and cosmic microwave background shift parameter. We used statistical minimization technique for the $\chi^{2}$ functions where we consider two different joint analyses (i) $\chi_{\Sigma_{2}}^{2}=\chi_{S N}^{2}+\chi_{H}^{2}+\chi_{B A O}^{2}$, and (ii) $\chi_{\text {tot }}^{2}=$ $\chi_{S N}^{2}+\chi_{H}^{2}+\chi_{B A O}^{2}+\chi_{C M B}^{2}$. The results of the analyses are presented in Table III.

We found that for $w_{d}=$ constant, the curvature parameter $\Omega_{k}$ plays significant role in the analysis. We investigated the cases $\Omega_{k} \neq 0$ and $\Omega_{k}=0$. The difference in the behavior of $\min \chi_{\Sigma}^{2}$ and $\min \chi_{\text {tot }}^{2}$ for both the variants has been presented in the top panels of Fig. 1 and in Table III. For the case $\Omega_{k} \neq 0$ of the model with $w_{d}=$ constant, the minimal value of $\chi_{\Sigma}^{2}$ is better, so for this case we presented two dimensional contour plots at $1 \sigma, 2 \sigma, 3 \sigma$ confidence levels in Fig. 1 .

Further, the possibility of variable EoS in DE has been investigated with 5 different variants in Eqs. (16) - 21) for the present interaction. We found that the variants may experience singularities (35) (see Fig. 2) at finite time, and hence, we excluded the domains of the parameters leading to the singular behavior and analyzed them by the current data sets mentioned above. In most of the cases we notice that one of the coupling parameters of the interaction possesses negative sign, so during the evolution of the universe the present interaction $Q$ changes its sign, thereby the direction of energy flow changes. This effect has been shown in Fig. 4 (see the bottom one) for some successful variants of the model. This shows that for $z \lesssim 0.4$, almost all successful variants (except Ansatz I) predict the flow of energy from CDM to DE (i.e. $Q<0$ ) while for $z \gtrsim 0.4$, the energy flows from $\mathrm{DE}$ to $\operatorname{CDM}$ (i.e. $Q>0$ ).

Furthermore, in figures 3, 5, we have presented the graphical variation of the $\min \chi_{\Sigma}^{2}$ and $\min \chi_{\text {tot }}^{2}$ over the model parameters for the variable EoS in DE presented in the paper. For Ansatz I and Ansatz IV (CPL parametrization), we have presented the contour plots in the two dimensional plane for several couple of model parameters at $1 \sigma, 2 \sigma, 3 \sigma$ confidence levels.

For all variants of the model with variable $w_{d}$, the positions of the minimum points in parameter spaces are essentially different for the functions $\chi_{\Sigma}^{2}$ and $\chi_{t o t}^{2}$. In fact, we observe that the minimal values of $\chi_{t o t}^{2}$ for these variants are larger in these cases. Based on the analysis we may conclude that the interacting model with $w_{d}=$ constant, is the most successful one in respect to all observational data.

Finally, some of the successful variants of the interaction model, such as, $w_{d}=$ constant (with $\Omega_{k}=0$ and $\Omega_{k} \neq 0$ ), Ansatz I, Ansatz IV, Ansatz V have been compared with some known non-interacting cosmological models, such as, $\Lambda$ CDM model, unified models, namely, the generalized Chaplygin gas (GCG), modified Chaplygin gas (MCG), a fluid with quadratic equation of state, and finally with CPL and linear parametrizations in DE. The results have been presented in Table IV.

It is found that the present interacting DE model with constant $w_{d}$ slightly favors the phantom region in agreement with the latest report [30]. The best absolute value of $\min \chi_{\Sigma}^{2}$ is achieved for the interacting model with EoS 21) (Ansatz V). The second result demonstrates that among the non-interacting models the model with quadratic EoS in 38 provides a better fit with the observational data. However, the number of model parameters of this non-interacting model $\left(N_{p}=5\right)$ is less than the number of model parameters for the interacting model 21), but larger than the number of model parameters in models GCG or $\Lambda$ CDM. Finally, we notice that although different models have different model parameters, still from AIC and BIC analysis, the models presented in Table IV do not deviate so much from the $\Lambda$ CDM model with minimum number of model parameters in comparison with others.

\section{ACKNOWLEDGMENTS}

The authors thank the referee for some essential comments to improve the work. SP was supported by the Science and Engineering Research Board through NPDF (File No: PDF/2015/000640).
[1] Riess A. G. et al., 1998, AJ, 116, 1009, arXiv: astro$\mathrm{ph} / 9805201$.

[2] Perlmutter S. et al., 1999, ApJ, 517, 565, arXiv:astro$\mathrm{ph} / 9812133$

[3] Percival W. J. et al., 2001, MNRAS, 327, 1297, arXiv:astro-ph/0105252

[4] Spergel D. N. et al., 2003, ApJS, 148, 175, arXiv:astro- $\mathrm{ph} / 0302209$

[5] Tegmark M. et al., 2004, Phys. Rev. D, 69, 103501, arXiv:astro-ph/0310723

[6] Eisenstein D. J. et al., 2005, ApJ, 633, 560, arXiv: astro-ph/0501171.

[7] Komatsu E. et al., 2011, ApJS, 192, 18, arXiv:1001.4538 [astro-ph.CO]. 
[8] Weinberg S., 1989, Rev. Mod. Phys., 61, 1

[9] Steinhardt P. J., 2003, Phil. Trans. Roy. Soc. Lond. A., 361, 2497

[10] Amendola L., Tsujikawa S., Dark Energy: Theory and Observations, Cambridge University Press, Cambridge UK (2010).

[11] Wetterich C., 1995, A\&A, 301, 321, arXiv:hepth/9408025

[12] Amendola L., 2000, Phys. Rev. D, 62, 043511, arXiv:astro-ph/9908023.

[13] Billyard A. P., Coley A. A., 2000, Phys. Rev. D, 61, 083503, arXiv:astro-ph/9908224

[14] Zimdahl W., Pavón D., Chimento L. P., 2001, Phys. Lett. B, 521, 133, arXiv:astro-ph/0105479

[15] Amendola L., Quercellini C., 2003, Phys. Rev. D, 68, 023514, arXiv:astro-ph/0303228

[16] Herrera R., Pavón D., Zimdahl W., 2004, Gen. Relt. Grav. 36, 2161, arXiv: astro-ph/0404086

[17] Chimento L. P., Jakubi A. S., Pavón D., Zimdahl W., 2003, Phys. Rev. D, 67, 083513, arXiv:astro$\mathrm{ph} / 0303145$

[18] He J.-H., Wang B., 2008 JCAP, 0806, 010, arXiv:0801.4233 [astro-ph].

[19] Quartin M. et al., 2008, JCAP, 0805, 007, arXiv:0802.0546 [astro-ph].

[20] Boehmer C. G., Caldera-Cabral G., Lazkoz R., Maartens R., 2008, Phys. Rev. D, 78, 023505, arXiv: 0801.1565 [gr-qc].

[21] Caldera-Cabral G., Maartens R., Urena-Lopez L. A., 2009, Phys. Rev. D, 79, 063518, arXiv: 0812.1827 [grqc].

[22] Chimento L. P., 2010, Phys. Rev. D, 81, 043525, arXiv: 0911.5687 [astro-ph.CO].

[23] Paliathanasis A., Tsamparlis M., 2014, Phys. Rev. D, 90, 043529

[24] Nunes R. C., Barboza E. M., 2014, Gen. Rel. Grav., 46, 1820, arXiv:1404.1620 [astro-ph.CO].

[25] Yang W., Xu L., 2014, Phys. Rev. D, 89, 083517

[26] Yang W., Xu L., 2014, Phys. Rev. D, 90, 083532

[27] Yang W., Li H., Wu Y., Lu J., 2016, JCAP, 10, 007

[28] Solà J., Perez J. d. C., Gómez-Valent A., Nunes R. C., 2016, arXiv:1606.00450 [gr-qc].

[29] Salvatelli V., Said N., Bruni M., Melchiorri A., Wands D., 2014, Phys. Rev. Lett, 113, 181301

[30] Nunes R. C., Pan S., Saridakis E. N., Phys. Rev. D, 94, 023508 (2016), arXiv:1605.01712 [astro-ph.CO].

[31] Kumar S., Nunes R. C., 2016, Phys. Rev. D, 94, 123511

[32] Kumar S., Nunes R. C., 2017, arXiv:1702.02143 [astroph.CO]

[33] van de Bruck C., Mifsud J., Morrice J., 2017, Phys. Rev. D, 95, 043513

[34] Pourtsidou A., Tram T., 2016, Phys. Rev. D, 94, 043518

[35] Di Valentino E., Melchiorri A., Mena O., 2017, Phys. Rev. D 96, 043503

[36] Xia D-M., Wang S., 2016, MNRAS, 463, 952

[37] Zimdahl W., 2005, Int. J. Mod. Phys. D, 14, 2319

[38] Wang B, Zang J, Lin C. -Y., Abdalla E., Micheletti S., 2007, Nucl. Phys. B., 778, 69

[39] Caldwell R. R., 2002, Phys. Lett. B, 545, 23, arXiv:astro-ph/9908168.

[40] Cheng C., Huang Q. -G., 2014, Phys. Rev. D, 89, 043003, arXiv:1306.4091 [astro-ph.CO].

[41] Planck Collaboration, 2014, A\&A, 571, A16, arXiv:1303.5076 [astro-ph.CO].
[42] Planck Collaboration, 2015, A\&A, 594, A13

[43] Rest A. et al., 2014, ApJ, 795, 44, arXiv:1310.3828 [astro-ph.CO].

[44] Shafer D. L., Huterer D., 2014, Phys. Rev. D, 89, 063510, arXiv:1312.1688 [astro-ph.CO].

[45] Mohseni Sadjadi H., Honardoost M., 2007, Phys. Lett. B, 647, 231, arXiv:gr-qc/0609076

[46] Pan S., Chakraborty S., 2014, Int. J. Mod. Phys. D, 23, 1450092, arXiv:1410.8281 [gr-qc].

[47] Bolotin Y. L., Kostenko A., Lemets O.A., Yerokhin D.A., 2014, Int. J. Mod. Phys. D, 24, 1530007, arXiv:1310.0085 [astro-ph.CO].

[48] Wang B., Abdalla E., Atrio-Barandela F., Pavón D., 2016, Rept. Prog. Phys., 79, 096901, arXiv:1603.08299 [astro-ph.CO].

[49] Pan S., Bhattacharya S., Chakraborty S., 2015, MNRAS, 452, 3038, arXiv:1210.0396 [gr-qc].

[50] Barris B. J. et al. 2004, ApJ, 602, 571, arXiv: astro$\mathrm{ph} / 0310843$

[51] Tonry J. L. et al., 2003, ApJ 594, 1, arXiv: astro$\mathrm{ph} / 0305008$

[52] Wang J. S., Wang F.Y., 2014, A\&A, 564, A137

[53] Chevallier M., Polarski D., 2001, Int. J. Mod. Phys. D, 10, 213, arXiv:gr-qc/0009008.

[54] Linder E. V. , 2003, Phys. Rev. Lett., 90, 091301, arXiv:astro-ph/0208512

[55] Riess A. G. et al., 2007, ApJ, 659, 98, arXiv: astro$\mathrm{ph} / 0611572$

56] Astier P., 2001, Phys. Lett. B, 500, 8, arXiv:astro$\mathrm{ph} / 0008306$

[57] Cooray A. R., Huterer D., 1999, ApJ, 513, L95, arXiv:astro-ph/9901097

[58] Weller J., Albrecht A., 2002, Phys. Rev. D, 65, 103512, arXiv:astro-ph/0106079

[59] Suzuki N. et al., 2012, ApJ, 746, 85, arXiv:1105.3470 [astro-ph.CO].

[60] Simon J., Verde L., Jimenez R., 2005, Phys. Rev. D, 71, 123001, arXiv:astro-ph/0412269

[61] Stern D., Jimenez R., Verde L., Kamionkowski M., Stanford S. A., 2010, JCAP, 1002, 008, arXiv:0907.3149 [astro-ph.CO].

[62] Moresco M. et al., 2012, JCAP, 1208, 006, arXiv:1201.3609 [astro-ph.CO].

[63] Zhang C. et al., 2014, Res. Astron. Astrophys., 14, 1221, arXiv:1207.4541 [astro-ph.CO].

[64] Moresco M., 2015, MNRAS, 450 L16, arXiv:1503.01116 [astro-ph.CO].

[65] Moresco M. et al., 2016, JCAP, 1605, 014, arXiv:1601.01701 [astro-ph.CO].

[66] Gaztañaga E., Cabre A., Hui L., 2009, MNRAS, 399, 1663, arXiv:0807.3551 [astro-ph].

[67] Blake C. et al., 2012, MNRAS, 425, 405

[68] Busca N. G. et al., 2013, A\&A, 552, A96

[69] Chuang C. -H., Wang Y., 2013, MNRAS, 435, 255, arXiv:1209.0210 [astro-ph.CO].

[70] Chuang C. -H. et al., 2013, MNRAS, 433, 3559, arXiv:1303.4486 [astro-ph.CO].

[71] Anderson L. et al., 2014, MNRAS, 439, 83, arXiv:1303.4666 [astro-ph.CO].

[72] Anderson L. et al., 2014, MNRAS, 441, 24, arXiv:1312.4877 [astro-ph.CO].

[73] Oka A. et al., 2014, MNRAS, 439, 2515, arXiv:1310.2820 [astro-ph.CO].

[74] Font-Ribera A. et al., 2014, JCAP, 1405, 027, 
arXiv:1311.1767 [astro-ph.CO].

[75] Delubac T. et al., 2015, A\&A, 574, A59, arXiv:1404.1801 [astro-ph.CO].

[76] Percival W. J. et al., 2010, MNRAS, 401, 2148, arXiv:0907.1660 [astro-ph.CO].

[77] Kazin E. A. et al., 2010, ApJ, 710, 1444, arXiv:0908.2598 [astro-ph.CO].

[78] Beutler F. et al., 2011, MNRAS, 416, 3017, arXiv:1106.3366 [astro-ph.CO].

[79] Blake C. et al., 2011, MNRAS, 418, 1707, arXiv:1108.2635 [astro-ph.CO].

[80] Padmanabhan N. et al., 2012, MNRAS, 427, 2132, arXiv:1202.0090 [astro-ph.CO].

[81] Seo H.-J. et al., 2012, ApJ, 761, 13, arXiv:1201.2172 [astro-ph.CO].

[82] Kazin E. A. et al., 2014, MNRAS, 441, 3524, arXiv:1401.0358 [astro-ph.CO].

[83] Ross A. J. et al., 2015, MNRAS, 449, 835, arXiv:1409.3242 [astro-ph.CO].

[84] Hinshaw G. et al., 2013, ApJS, 208, 19, arXiv:1212.5226 [astro-ph.CO].

[85] Huang Q.-G., Wang K., Wang S., 2015, JCAP, 1512, 022, arXiv:1509.00969 [astro-ph].

[86] Nesseris S., Perivolaropoulos L., 2005, Phys. Rev. D, 72, 123519, arXiv:astro-ph/0511040.

[87] Conley A. et al., 2011, ApJS, 192, 1, arXiv:1104.1443 [astro-ph.CO].

[88] Ruiz E.J., Shafer D.L., Huterer D., Conley A., 2012, Phys. Rev. D, 86, 103004, arXiv:1207.4781 [astroph.CO].
[89] Aubourg E. et al., 2015, Phys. Rev. D, 92, 123516, arXiv:1411.1074 [astro-ph.CO].

[90] G. S. Sharov, JCAP, 06, 023 (2016), arXiv:1506.05246 [gr-qc].

[91] Nojiri S., Odintsov S. D., Tsujikawa S., Phys. Rev. D, 71, 063004 (2005), hep-th/0501025.

[92] Nojiri S., Odintsov S. D., Phys. Lett. B, 636, 44 (2010), arXiv:0911.2781.

[93] Bamba K., Capozziello S., Nojiri S., Odintsov S. D., Astrophys. Sp. Sc, 342, 155 (2012), arXiv:1205.3421] [gr$\mathrm{qc}]$.

[94] H. Akaike, IEEE Transactions of Automatic Control 19, 716 (1974)

[95] Szydłowski M., Kurek A., Krawiec A., 2006, Phys. Lett. B 642, 171, arXiv:astro-ph/0604327.

[96] Shi K., Huang Y. F., Lu T., 2012, MNRAS, 426, 2452, arXiv:1207.5875 [astro-ph.CO]

[97] He J.-H., Wang B., Abdalla E., 2008, Phys. Lett. B, 671, 139, arXiv:0807.3471 [gr-qc].

[98] Valiviita J., Majerotto E., Maartens R., 2008, JCAP, 0807, 020, arXiv: 0804.0232 [astro-ph].

[99] Debnath U., Banerjee A., Chakraborty S., 2004, Class. Quant. Grav. 21, 5609, arXiv:gr-qc/0411015

[100] Benaoum H. B., 2002, arXiv:hep-th/0205140.

[101] Kamenshchik A. Y., Moschella U., Pasquier V., 2001, Phys. Lett. B, 511, 265, arXiv:gr-qc/0103004.

[102] Ananda K. N., Bruni M., 2006, Phys. Rev. D, 74, 023523, arXiv:astro-ph/0512224 